\title{
Signatures of top flavour-changing dark matter
}

\author{
Jorgen D'Hondt, ${ }^{a}$ Alberto Mariotti, ${ }^{a}$ Kentarou Mawatari, ${ }^{a, b}$ Seth Moortgat, ${ }^{a}$ \\ Pantelis Tziveloglou ${ }^{a}$ and Gerrit Van Onsem ${ }^{a, c}$ \\ ${ }^{a}$ Theoretische Naturkunde and IIHE/ELEM, Vrije Universiteit Brussel, \\ International Solvay Institutes, \\ Pleinlaan 2, B-1050 Brussels, Belgium \\ ${ }^{b}$ Laboratoire de Physique Subatomique et de Cosmologie, Université Grenoble-Alpes, CNRS/IN2P3, \\ 53 Avenue des Martyrs, F-38026 Grenoble, France \\ ${ }^{c} D E S Y$, \\ Notkestr. 85, D-22607 Hamburg, Germany \\ E-mail: Jorgen.DHondt@vub.ac.be, amariotti.vub@gmail.com, \\ kentarou.mawatari@lpsc.in2p3.fr, seth.moortgat@gmail.com, \\ pantelis.tziveloglou@gmail.com, gerrit.van.onsem@desy.de
}

ABSTRACT: We develop the phenomenology of scenarios in which a dark matter candidate interacts with a top quark through flavour-changing couplings, employing a simplified dark matter model with an $s$-channel vector-like mediator. We study in detail the top-charm flavour-changing interaction, by investigating the single top plus large missing energy signature at the LHC as well as constraints from the relic density and direct and indirect dark matter detection experiments. We present strategies to distinguish between the top-charm and top-up flavour-changing models by taking advantage of the lepton charge asymmetry as well as by using charm-tagging techniques on an extra jet. We also show the complementarity between the LHC and canonical dark matter experiments in exploring the viable parameter space of the models.

Keywords: Beyond Standard Model, Cosmology of Theories beyond the SM

ArXiv EPRINT: 1511.07463 


\section{Contents}

1 Introduction 1

2 Models 3

2.1 Simplified models 3

2.2 Top-up vs. top-charm interactions 8

$\begin{array}{ll}2.3 \text { Effective field theory description } & 9\end{array}$

3 Signatures 11

3.1 Monotop at the LHC 11

3.1.1 Prospects for the LHC Run-II 12

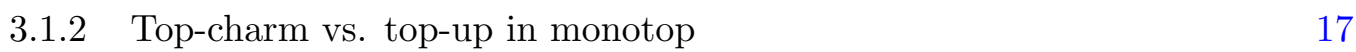

$\begin{array}{lll}3.2 & \text { Canonical dark matter searches } & 19\end{array}$

3.3 Complementarity between the LHC and non-collider experiments 22

4 Conclusions and discussions $\quad 24$

\section{Introduction}

The description of dark matter (DM), whose abundant presence in the universe is supported by overwhelming observational evidence, is nowadays one of the main motivations for physics beyond the Standard Model (SM). The usual paradigm to realize the correct relic abundance of DM in the universe relies on a weak coupling between the SM particles and the DM candidate, the so-called weakly interacting massive particle (WIMP). This scenario implies that signatures of DM could be discovered in colliders and canonical DM experiments, such as direct and indirect detection experiments. Indeed, in the last years there has been intense activities in developing DM searches at the LHC and their possible interplay with underground and satellite DM experiments.

Since the properties and the interaction of DM with SM particles are unknown, a current bottom-up approach is to employ simplified models of DM that capture the phenomenology of different types of theories beyond the SM. Simplified models typically consist of new DM species and mediator fields that connect the SM and the DM sectors. They are usually parametrised by the masses of the mediator and DM fields as well as the couplings of the mediator to the DM and the SM particles (see e.g. [1]).

One interesting possibility among various types of simplified models is that the DM sector couples to the SM through flavour non-universal interactions. In this context, one can envisage simplified DM models with flavour violating structures and identify the relevant constraints as well as the possible novel signatures of such scenarios. Non-universal flavour 
couplings can arise in $Z^{\prime}$ models [2-4] and in the flavoured DM paradigm [5-14], where the DM candidate belongs to a sector which is also responsible to explain the flavour structure in the SM.

Among the non-universal flavour interactions, the couplings that involve third generation quarks are less constrained by low-energy experiments. Moreover, the top quark, being the heaviest of the SM particles, can be an interesting candidate to represent the portal through which DM couples to the SM sector. The LHC signatures and the DM constraints on such models are in general different from those of the usual WIMP models. Flavour non-universal yet diagonal DM models characterised by the four-fermion interaction between a DM pair and a top-quark pair have been studied in an effective field theory (EFT) approach [15-17] and searched for in a top-quark pair plus missing energy final state at the LHC Run-I $[18,19]$. Recently more studies in simplified models involving top quarks have been done [20-23].

It is interesting to extend these studies to a possibility of having a flavour-changing coupling involving the top quarks. Inclusion of the top flavour-changing interaction with DM opens a peculiar collider signature, the so-called monotop signature [3, 9, 24], already searched for by the ATLAS and CMS Collaborations [25, 26]. We note that in the past the top-up flavour-changing coupling has been extensively studied [3, 4, 9, 24, 27-32], while the top-charm coupling has received less attention.

Moreover, top flavour-changing DM models are interesting also because they can account [33] for the excess of gamma rays originating in the centre of our galaxy [34-36], without any conflict with constraints from flavour physics. We note that there has been recently some debate about the DM origin of this excess and several alternative explanations have been put forward (see e.g. [37-40] and references therein). Nevertheless it is interesting, during the analysis of our simplified model, to discuss the tantalizing possibility that this is indeed due to a DM signal.

In this work, we study in detail the phenomenology of a simplified DM model with topcharm flavour-changing interactions, highlighting the difference from the top-up flavourchanging case. We present the prospects for the LHC Run-II, concentrating on the leptonic single-top final state. We also investigate the charge asymmetry of the lepton in the final state and charm-tagging techniques to distinguish between the top-charm and top-up interaction models. Apart from the LHC DM searches, we discuss in detail the relic density, and indirect and direct detection constraints on the model. The interplay for DM searches between the LHC and non-collider experiments will reveal different features between the top-charm flavour-changing interaction and the top-up one, furnishing another interesting manifestation of complementarity among different DM search experiments.

The paper is organized as follows. In section 2 we introduce the simplified DM model involving the top flavour-changing interaction, and classify the signatures depending on the model parameters. We also discuss the relation with the EFT approach. In section 3 we discuss signatures of our model at the LHC as well as in the relic density, direct and indirect detection experiments. Section 4 is devoted to our summary and discussion. 


\section{Models}

We start this section by describing simplified models for top flavour-changing DM and discussing the possible signatures. Then, we give some remarks about the similarities and differences of the signatures between the top-up and top-charm flavour-changing models. Lastly, we introduce the corresponding EFT description to briefly mention the relation with the simplified model and its validity.

\subsection{Simplified models}

Since the dynamics of DM are not known, simplified models have been proposed that parametrise the way DM interacts with SM particles. In the simplest versions, the SM is extended by two new species, a DM particle and a particle that mediates the interaction between the DM and SM particles, called the "mediator". In this work we are interested in the phenomenological implications of the interactions of a fermionic Dirac DM $(\chi)$ with the quark sector through an $s$-channel vector-like mediator $\left(Z^{\prime}\right) .{ }^{1}$ The interaction Lagrangian is given by

$$
\mathcal{L}_{\text {int }}=g_{\chi} \bar{\chi} \gamma_{\mu} \chi Z^{\prime \mu}+\left(g_{i j}^{Q} \bar{Q}_{L}^{i} \gamma_{\mu} Q_{L}^{j} Z^{\prime \mu}+g_{i j}^{u} \bar{u}_{R}^{i} \gamma_{\mu} u_{R}^{j} Z^{\prime \mu}+g_{i j}^{d} \bar{d}_{R}^{i} \gamma_{\mu} d_{R}^{j} Z^{\prime \mu}+\text { h.c. }\right) .
$$

As mentioned in section 1, we are interested in flavour-changing DM interactions in the up-quark sector, specifically involving the top quark. If the interactions involve the $\mathrm{SU}(2)_{L}$ doublets, large flavour off-diagonal couplings in the left-handed sector would imply large flavour violation also for the down sector, which are strongly constrained by flavour physics, e.g. by $B_{d}-\bar{B}_{d}$ mixing [42]. Instead, flavour-changing operators involving right-handed top quarks and up or charm quarks are phenomenologically viable. Therefore, we focus on studying the effective flavour-changing interaction with right-handed up-type quarks in the Lagrangian (2.1), i.e.

$$
\mathcal{L}_{\text {int }}=g_{\chi} \bar{\chi} \gamma^{\mu} \chi Z^{\prime \mu}+\left(g_{13} \bar{u}_{R} \gamma_{\mu} t_{R} Z^{\prime \mu}+g_{23} \bar{c}_{R} \gamma_{\mu} t_{R} Z^{\prime \mu}+\text { h.c. }\right) .
$$

Hereafter we omit the superscript ' $u$ ' of the coupling parameters $g_{i j}^{u}$. Note that if both the up and charm flavour-changing operators are present we expect a relevant box diagram contribution to the $D_{0}-\bar{D}_{0}$ mixing [43]. Hence in the following we consider one of these operators at a time. With the above simplification, the model has in total four parameters, i.e. two couplings and two masses:

$$
\left\{g_{\chi}, g_{i 3}, m_{\chi}, m_{Z^{\prime}}\right\} \quad \text { with } \quad i=1 \text { or } 2 .
$$

Table 1 summarises the various expected signatures in different regions of the parameter space, which will be discussed below.

We note here that, since we intend to focus on the experimental signatures of the top flavour-changing DM model, we postpone to future investigation a detailed study of the possible UV completions. At the end of the paper we discuss the basic guidelines and the most relevant issues in constructing such complete theories, identifying possible parallel implications for low-energy phenomenology.

\footnotetext{
${ }^{1}$ An s-channel pseudo-scalar mediator case was studied recently in [41].
} 


\begin{tabular}{|cccccc|}
\hline & & DM annihilation & $\Delta \Gamma_{t}$ & $\Gamma_{Z^{\prime}}$ & monotop \\
\hline $2 m_{\chi}>m_{t}$ & $m_{Z^{\prime}}>2 m_{\chi}>m_{t}$ & yes & 0 & $t q, \chi \chi$ & yes \\
& $m_{Z^{\prime}} \sim 2 m_{\chi}>m_{t}$ & enhanced & 0 & $t q$ & suppressed \\
& $2 m_{\chi}>m_{Z^{\prime}}>m_{t}$ & yes & 0 & $t q$ & suppressed \\
& $2 m_{\chi}>m_{t}>m_{Z^{\prime}}$ & yes & $q Z^{\prime}$ & suppressed & suppressed \\
\hline$m_{t}>2 m_{\chi}$ & $m_{Z^{\prime}}>m_{t}>2 m_{\chi}$ & suppressed & rare $q \chi \chi$ & $t q, \chi \chi$ & yes \\
& $m_{t}>m_{Z^{\prime}}>2 m_{\chi}$ & suppressed & $q Z^{\prime}$ & $\chi \chi$ & yes \\
& $m_{t}>2 m_{\chi}>m_{Z^{\prime}}$ & suppressed & $q Z^{\prime}$ & suppressed & suppressed \\
\hline
\end{tabular}

Table 1. Signatures of the top flavour-changing DM model in each mass spectrum. $\Delta \Gamma_{t}$ and $\Gamma_{Z^{\prime}}$ represent the partial decay modes of the top quark and the $Z^{\prime}$, respectively.

DM annihilation. The annihilation of the DM candidate to SM particles during the early universe determines its thermal relic density while late time annihilation in the centre of galaxies offers a possibility to detect DM indirectly, via observation of its SM products. The annihilation channel is

$$
\chi+\bar{\chi} \rightarrow Z^{\prime(*)} \rightarrow t+\bar{q} \text { and } \bar{t}+q
$$

where $q$ denotes an up quark or a charm quark. The annihilation is kinematically efficient for $2 m_{\chi}>m_{t}$ and is enhanced by threshold effects for $m_{Z^{\prime}} \sim 2 m_{\chi}$. If $m_{t}>2 m_{\chi}$, the annihilation is suppressed since it occurs via the off-shell top quark (and/or $W$ boson). Therefore, DM would be overly produced in the early universe, inconsistent with the current observation of the relic abundance of DM. The relic density and the phenomenology of indirect detection experiments are detailed in section 3.2.

DM-nucleus scattering. Another process that is potentially relevant for the phenomenology of our DM models is the elastic scattering

$$
\chi+N \rightarrow \chi+N
$$

of DM particles off nuclei of direct detection experiments. Detection of DM in this type of experiments is based on the observation of the nuclear recoil energy that the scattering releases. Different from usual flavour-conserving DM models, the above interaction in our model occurs only through loop diagrams, and hence it is expected to be strongly suppressed. In section 3.2 we discuss further the direct detection phenomenology and calculate the one-loop process in our model.

Top decay. If $m_{t}>2 m_{\chi}$, top quarks can decay into a light quark (up or charm) plus a pair of DM through the on-shell or off-shell mediator:

$$
t \rightarrow q+Z^{(*)} \rightarrow q+\chi+\bar{\chi} .
$$

Figure 1(left) shows the total width of the top quark (top) and the branching ratio of the above anomalous decay (bottom) as a function of the mediator mass, where we assume a 

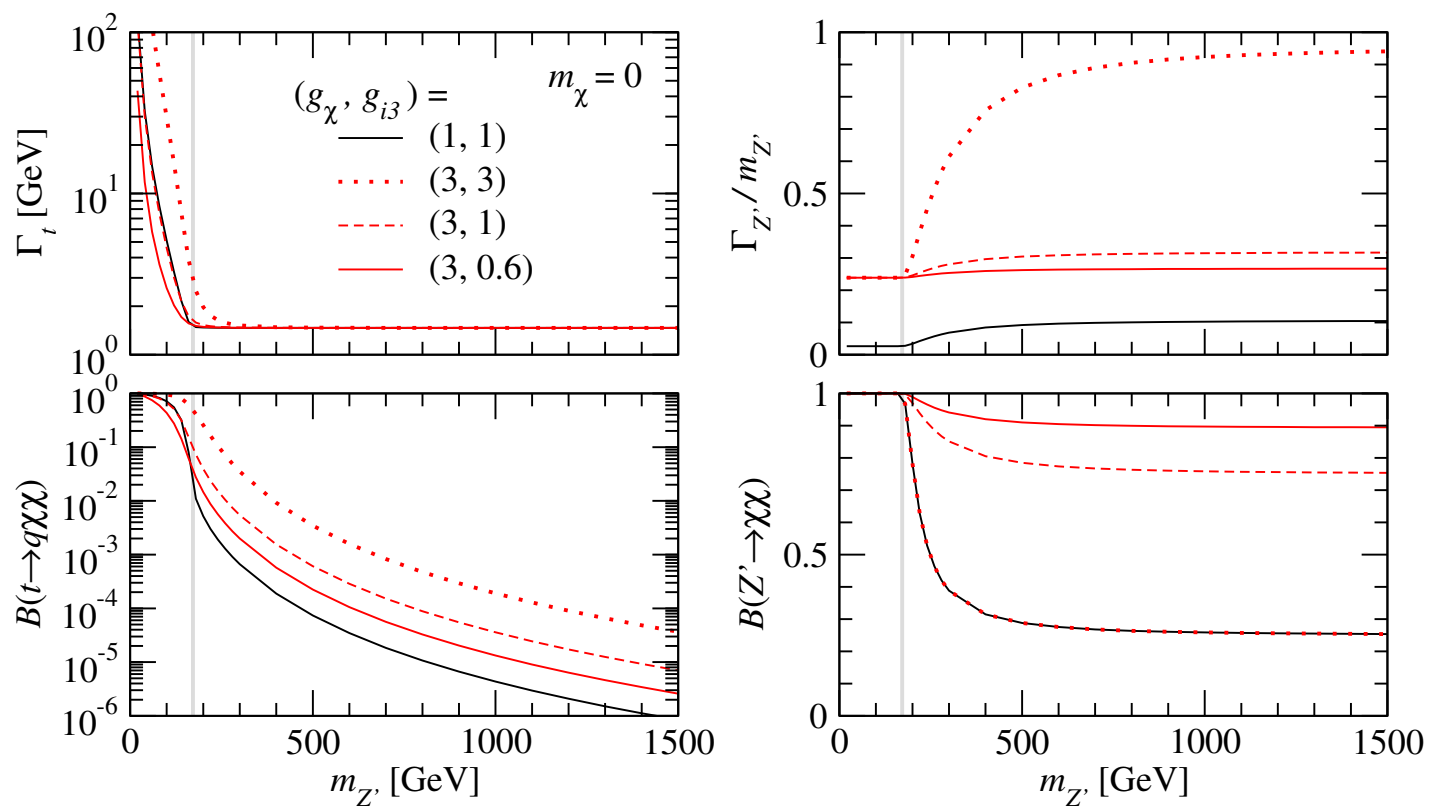

Figure 1. Left: top decay width and branching ratio of the anomalous top decay as a function of the mediator mass. Right: $\Gamma_{Z^{\prime}} / m_{Z^{\prime}}$ ratio and branching ratio of the mediator decay into a pair of DM as a function of the mediator mass. We assume a massless DM and take different values of the coupling parameters. The vertical grey line indicates $m_{Z^{\prime}}=m_{t}$.

massless DM. For $m_{Z^{\prime}}<m_{t}$, where the top quark decays into the mediator on mass-shell, the width becomes too broad to be consistent with the current bound $1 \lesssim \Gamma_{t} \lesssim 4 \mathrm{GeV}$ from Tevatron $[44,45]$. We note that in this parameter region the width depends only on the coupling $g_{i 3}$ and the mediator mass. For $m_{Z^{\prime}}>m_{t}$, on the other hand, the anomalous decay arises through the off-shell mediator and hence is strongly suppressed as the mediator mass increases, unless the couplings are very large. In figure 2(left), we show the DM mass dependence with the coupling fixed at $\left(g_{\chi}, g_{i 3}\right)=(3,0.6)$. The anomalous decay becomes smaller when the DM becomes heavier due to the phase space suppression.

As a reference, we mention the current limit on the top-quark flavour-changing neutral current (FCNC) decay, although the analyses have not considered missing energy. The current most stringent limit is for the $t \rightarrow Z q$ mode as $B(t \rightarrow Z q)<5 \times 10^{-4}$ from the LHC Run-I data [46].

Mediator decay. The decay of the mediator depends strongly on the mass spectra and the values of the couplings. There are two decay modes, with partial widths given by

$$
\begin{aligned}
\Gamma\left(Z^{\prime} \rightarrow \chi \bar{\chi}\right) & =\frac{g_{\chi}^{2} m_{Z^{\prime}}}{12 \pi} \sqrt{1-\frac{4 m_{\chi}^{2}}{m_{Z^{\prime}}^{2}}}\left(1+2 \frac{m_{\chi}^{2}}{m_{Z^{\prime}}^{2}}\right), \\
\Gamma\left(Z^{\prime} \rightarrow t \bar{q} / \bar{t} q\right) & =\frac{g_{i 3}^{2} m_{Z^{\prime}}}{4 \pi}\left(1-\frac{m_{t}^{2}}{m_{Z^{\prime}}^{2}}\right)\left(1-\frac{m_{t}^{2}}{2 m_{Z^{\prime}}^{2}}-\frac{m_{t}^{4}}{2 m_{Z^{\prime}}^{4}}\right) .
\end{aligned}
$$

Figures 1 and 2(right) show the ratio of the width to the mass for the mediator (top) and the branching ratio of the mediator decay into a pair of DM (bottom) as a function 

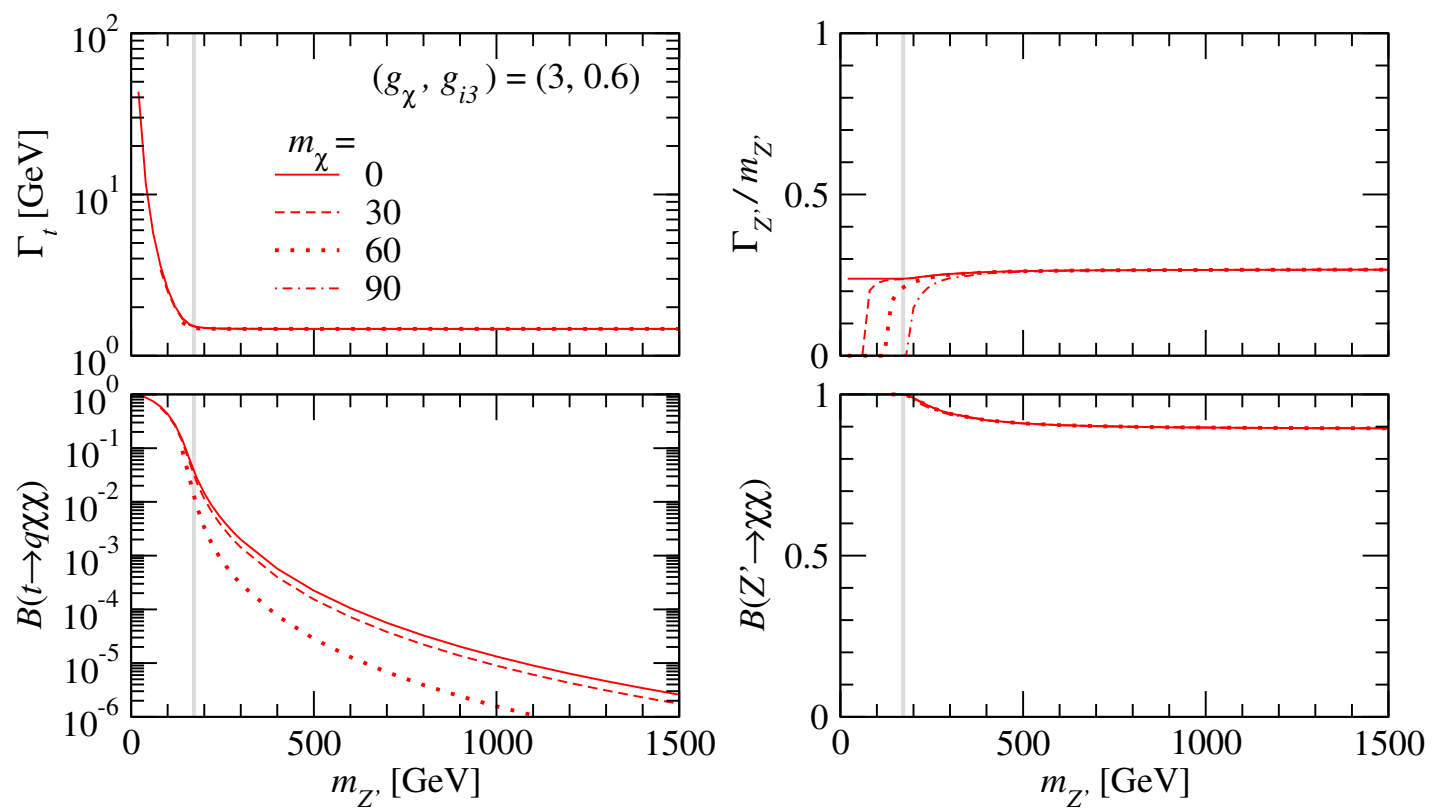

Figure 2. Same as figure 1, but for the different DM masses. The coupling parameters are fixed at $\left(g_{\chi}, g_{i 3}\right)=(3,0.6)$.

of the mediator mass. For the massless DM (figure 1), if $m_{Z^{\prime}}<m_{t}$, the mediator can only decay into a pair of DM. For $m_{Z^{\prime}} \gg m_{t}$, on the other hand, the mediator dominantly decays into a top quark and a light quark if the two couplings are of similar size $g_{\chi} \sim g_{i 3}$ due to the colour factor. For a large $g_{i 3}$ coupling the width becomes too large. Figure 2(right) shows that the branching ratio does not depend on the DM mass for $m_{Z^{\prime}} \gg 2 m_{\chi}$. We note that, even if $Z^{\prime}$ is in the bottom of the mass spectrum, it decays via the off-shell top quark (and/or $W$ boson), but its decay is strongly suppressed. Moreover, the loop-induced dijet decay channel can be relevant [4].

In this work, we are interested in the DM signature at the LHC, and hence we take $g_{\chi}=5 \times g_{i 3}$ so that the branching ratio $B\left(Z^{\prime} \rightarrow \chi \bar{\chi}\right)$ becomes more than 0.9 . As our illustrative benchmark point, we take

$$
g_{\chi}=3.0 \quad \text { and } \quad g_{i 3}=0.6
$$

which can provide reasonable signal rate at the LHC as we will show below, still keeping the mediator width as $\Gamma_{Z^{\prime}} / m_{Z^{\prime}} \lesssim 1 / 4$.

Collider signatures. A distinctive collider signature in our model is a single top-quark production in association with large missing energy, the so-called monotop signature:

$$
p+p \rightarrow t+Z^{\prime(*)} \rightarrow t+\chi+\bar{\chi}
$$

where $t$ denotes a top quark or a top anti-quark. The Feynman diagrams are shown in figure 3 (top).

Figure 4 shows the total cross sections of $p p \rightarrow t \chi \bar{\chi}$ (solid lines) for our benchmark point (2.9) at $\sqrt{s}=8$ and $13 \mathrm{TeV}$ as a function of the mediator mass, where we fix the DM 

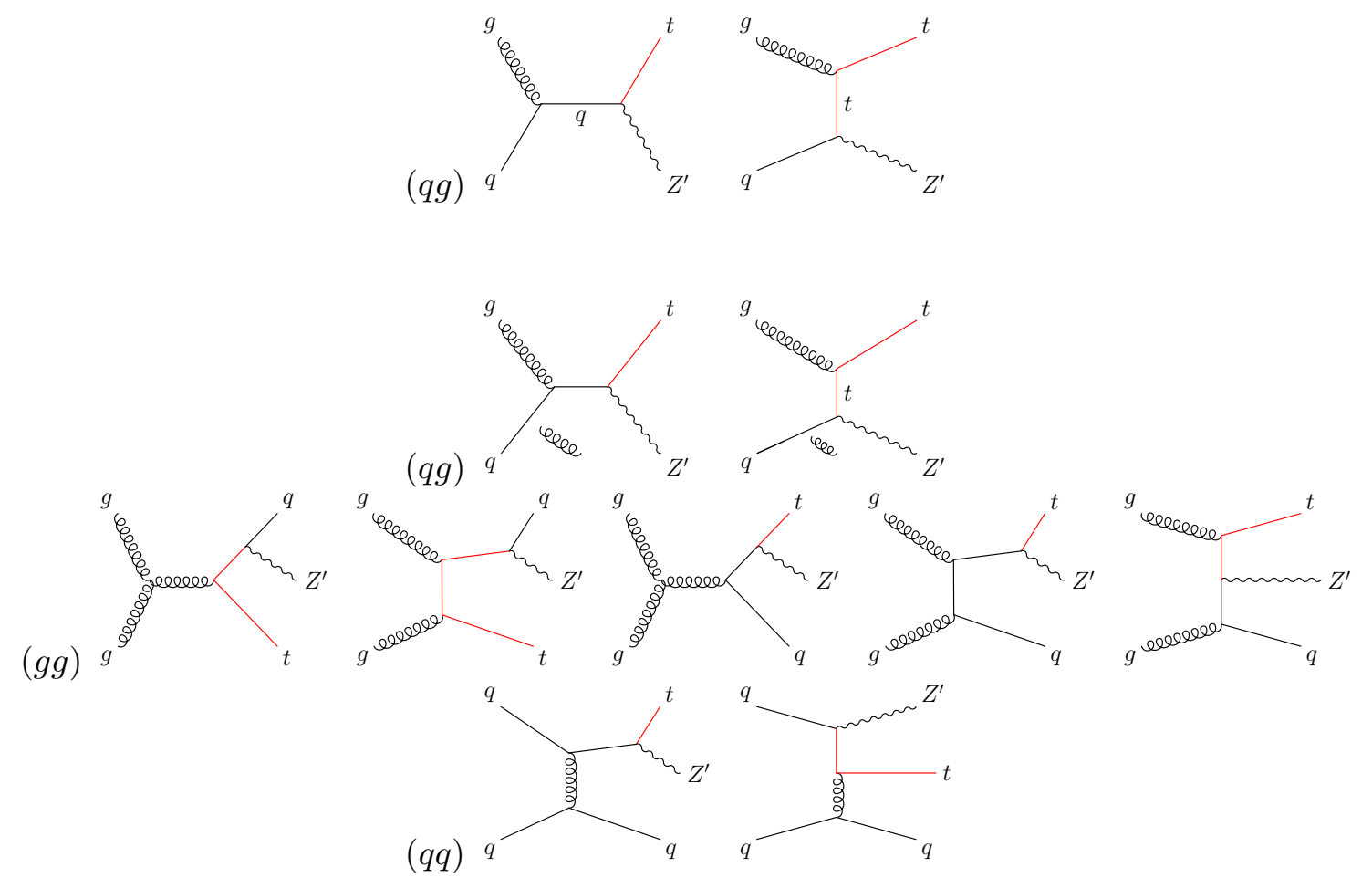

Figure 3. Representative Feynman diagrams for $p p \rightarrow t Z^{\prime}$ (top) and $p p \rightarrow t Z^{\prime} j$ (bottom).
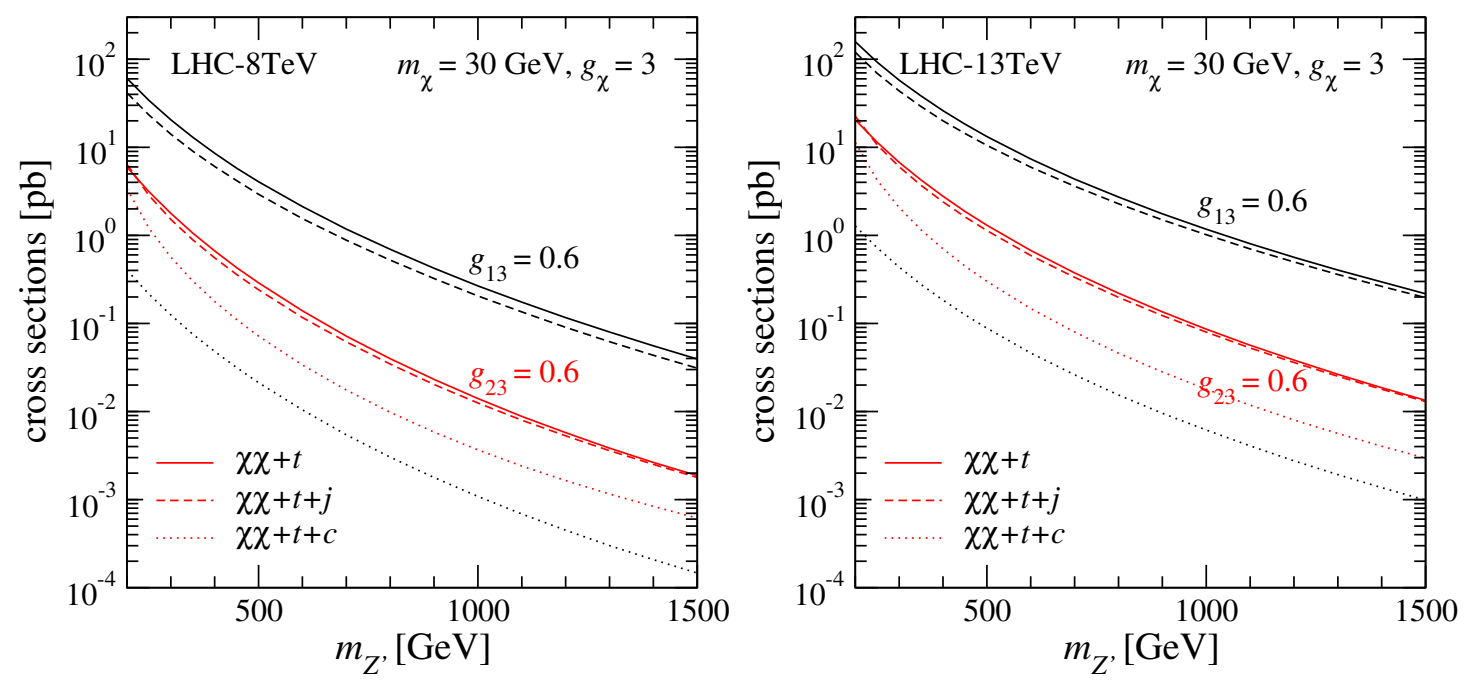

Figure 4. Total cross sections for DM pair production in association with a top quark in $p p$ collisions at $\sqrt{s}=8 \mathrm{TeV}$ (left) and $13 \mathrm{TeV}$ (right) as a function of the mediator mass, where we assume the top-up (black) and top-charm (red) flavour-changing DM model and fix the DM mass at $30 \mathrm{GeV}$. For the processes with a $(c-)$ jet, the kinematical cuts $p_{T}^{j}>25 \mathrm{GeV}$ and $\left|\eta^{j}\right|<2.5$ are imposed. 
mass at $30 \mathrm{GeV}$ and consider only $m_{Z^{\prime}}>m_{t}$ to avoid the large top width. The cross section in the top-up model is larger than that in the top-charm one roughly by a factor of 10 , simply explained by the difference between the up and charm parton distribution functions (PDFs). The cross sections for both models increase by $3-5$ times from $\sqrt{s}=8 \mathrm{TeV}$ to $13 \mathrm{TeV}$. We note that the cross sections do not depend on the DM mass as long as the mediator is produced on-shell and the $Z^{\prime} \rightarrow \chi \bar{\chi}$ branching ratio is fixed.

In figure 4 , we also show the cross sections for the monotop process in association with a jet by dashed lines, where we impose the transverse momentum $p_{T}^{j}>25 \mathrm{GeV}$ and the pseudorapidity $\left|\eta^{j}\right|<2.5$ as minimal cuts. As extra QCD jets often emerge at the energy scale of the LHC, we should take them into account for a reliable prediction, and indeed the cross sections are comparable with the ones without an additional jet, especially for the $\sqrt{s}=13 \mathrm{TeV}$ case. As shown in figure 3 , in addition to the leading-order (LO) $q g \rightarrow t Z^{\prime}$ process with a gluon emission, the $g g$ and $q q$ initial states contribute and enhance the production rate. We note that the steeper fall of the $\chi \bar{\chi} t j / c$ cross sections in the topcharm model for around $m_{Z^{\prime}} \sim 200 \mathrm{GeV}$ comes from the top-pair contribution with the anomalous top decay, i.e. $\sigma(t \bar{t}) \times B(t \rightarrow q \chi \bar{\chi})$; see also figure 1(bottom-left).

The extra jet contribution can not only enhance the signal but also give some hint to distinguish between the top-up and top-charm models. Although the charm-quark tagging is more difficult than the bottom-quark tagging, the technique is under development both in the ATLAS and CMS collaborations and promising for the LHC Run-II. Assuming an ideal $100 \% c$-tagging efficiency, the dotted lines in figure 4 present the cross sections for a single top plus a charm jet in association with missing transverse energy. The production cross section in the top-up model is not zero, but strongly suppressed, since this comes from the $u c$ initial state only. For the top-charm model, on the other hand, the $g g$ scattering can provide the charm final state, and hence the production rate does not decrease so much even after identifying the jet as a charm jet. In this work, therefore, we take into account extra jets for the monotop signal by employing a matrix-element parton-shower (ME+PS) merging scheme [47] and investigate if we can get additional information on the models.

It should be noted that if $g_{\chi} \lesssim g_{i 3}$, i.e. if the DM interaction with $Z^{\prime}$ is subdominant, the dark sector is essentially decoupled and the model becomes a type of non-universal $Z^{\prime}$ model, such as those intensively discussed in the context of the top forward-backward asymmetry reported by Tevatron $[48,49]$. The $t$-channel $Z^{\prime}$ produces top quarks in the forward region for $q \bar{q} \rightarrow t \bar{t}[2]$. Another distinctive signature in this scenario is same-sign $t t$ pair production via $q q$ or $\bar{q} \bar{q}$ scattering with a $t$-channel $Z^{\prime}[50,51]$, searched for already in the LHC Run-I data $[52,53]$. The diagrams in figure 3 also produce the same-sign top pair with jets if the $Z^{\prime}$ dominantly decays into a top and a light quark. Note that if the new vector boson is not self-conjugate the model does not lead to the same-sign top signal [54].

\subsection{Top-up vs. top-charm interactions}

As mentioned in section 1, we focus primarily on the less explored top-charm DM model. Here, we list certain remarks related to the similarities and differences of the top-up and top-charm flavour-changing DM models, that will be discussed along the paper: 
- The annihilation of DM (relevant for the calculation of the relic abundance and the indirect detection limits) is practically the same in the top-up and top-charm DM models.

- DM direct detection physics is a priori different in the two models, since the top-up DM model involves the interaction with a valence quark in nucleons. However we will demonstrate that both the top-up and top-charm models are beyond the reach of current and near future direct detection experiments.

- The contribution to the top width and the mediator width is once again practically the same in the top-up and top-charm DM models.

- At the LHC, the main difference between the top-up and top-charm DM models lies in the monotop production cross section (if we assume $g_{13} \sim g_{23}$ ); see figure 4 .

- In the monotop signature, as we will explore in section 3.1, the top-up and top-charm DM models can be distinguished by lepton charge asymmetry and by a charm-tagging technique on an extra jet.

In conclusion, distinguishing between the top-up and top-charm models is very difficult in non-collider experiments, and may be challenging at the LHC. However, in this work we demonstrate that a combined approach can allow us in certain cases to select one of the two models as soon as a hint of new physics is discovered or enough luminosity at the LHC is collected.

\subsection{Effective field theory description}

Before turning to the detailed study of the phenomenology of the DM model, we introduce the corresponding EFT description to briefly mention the relation to the simplified model and its validity.

The EFT Lagrangian corresponding to the simplified model in (2.2) is given by the following four-fermion contact interaction operators

$$
\mathcal{L}_{\mathrm{int}}^{\mathrm{EFT}}=\frac{1}{\Lambda^{2}} \bar{\chi} \gamma^{\mu} \chi\left(c_{13} \bar{u}_{R} \gamma_{\mu} t_{R}+c_{23} \bar{c}_{R} \gamma_{\mu} t_{R}+\text { h.c. }\right),
$$

where $\Lambda$ is the cutoff scale.

The EFT Lagrangian provides a valid description of the simplified model in the limit where the mediator is much heavier than the energy scale probed by the experiment. Therefore, for low-energy processes such as the DM annihilation in the late universe (relevant for indirect DM searches) and the elastic scattering of DM off nuclei (relevant for direct DM searches), the EFT Lagrangian provides an accurate description of the dynamics. However, if the energy reach is comparable or higher than the mediator mass such as at the LHC, the EFT approach does not offer a suitable framework for describing the DM interactions [55-60].

In order to give an idea of the region of the EFT parameter space that the LHC explores, let us first show the branching ratio of $t \rightarrow q \chi \bar{\chi}(q=u$ or $c)$ in the EFT description 

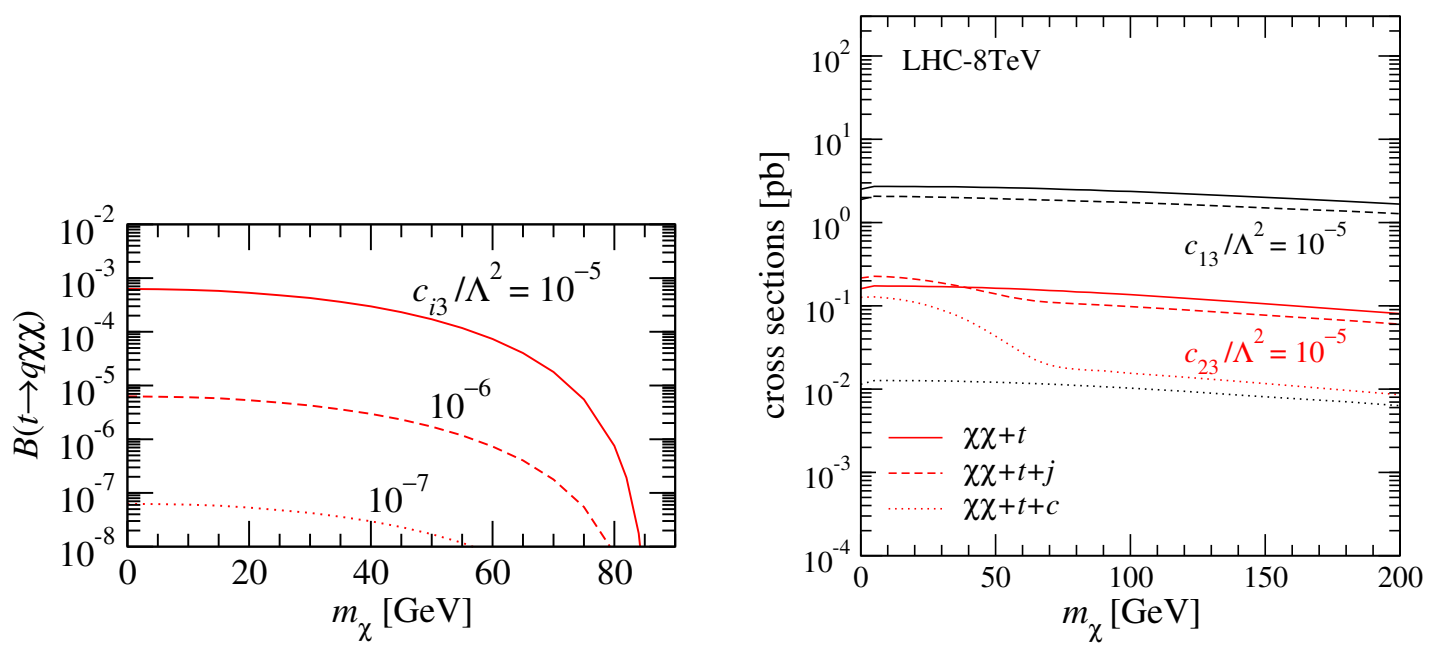

Figure 5. Left: branching ratio of the anomalous top decay as a function of the DM mass for different coupling parameters in the EFT description. Right: total cross sections for DM pair production in association with a top quark in $p p$ collisions at $\sqrt{s}=8 \mathrm{TeV}$ as a function of the DM mass, where we assume the top-up (black) and top-charm (red) flavour-changing DM model in the EFT description. For the processes with a (c-)jet, the kinematical cuts $p_{T}^{j}>25 \mathrm{GeV}$ and $\left|\eta^{j}\right|<2.5$ are imposed.

in figure 5(left), corresponding to the left-bottom panel in figures 1 and 2 for the simplified model. The partial width depends only on $\left(c_{i 3} / \Lambda^{2}\right)^{2}$ and $m_{\chi}$. For $m_{\chi} \geq m_{t} / 2$ the decay channel is kinematically closed. For $c_{i 3} / \Lambda^{2}=10^{-5}$ the anomalous decay branching ratio can be of the order of $10^{-3}-10^{-4}$.

Figure 5(right) shows the production rates for the single top plus missing energy at $\sqrt{s}=8 \mathrm{TeV}$ in the EFT description, corresponding to figure 4 (left). $c_{i 3} / \Lambda^{2}$ is fixed at $10^{-5}$. The cross sections are insensitive to the DM mass, except for the light DM case in the top-charm model, where the top-pair production contributes significantly.

As we will discuss, the monotop searches at the LHC Run-I set an upper limit cross section of about $\mathcal{O}(1) \mathrm{pb}[25,26]$. Hence the EFT parameter $c_{23} / \Lambda^{2} \sim \mathcal{O}\left(10^{-5}\right)$ is the range that the LHC can explore in this model. By the tree-level matching relation between the EFT coefficient $c_{i 3}$ and the $Z^{\prime}$ model parameters

$$
\frac{c_{i 3}}{\Lambda^{2}}=\frac{g_{\chi} g_{i 3}}{m_{Z^{\prime}}^{2}},
$$

we can translate the value of the EFT parameter to the simplified model obtaining

$$
m_{Z^{\prime}} \sim\left\{\begin{array}{lll}
300 \mathrm{GeV} & \text { with } & g_{\chi} g_{23} \sim \mathcal{O}(1), \\
1 \mathrm{TeV} & \text { with } & g_{\chi} g_{23} \sim \mathcal{O}(10), \\
10 \mathrm{TeV} & \text { with } & g_{\chi} g_{23} \sim \mathcal{O}\left(10^{3}\right) .
\end{array}\right.
$$

For reasonable values of the couplings, the on-shell production of the mediator is within the LHC reach, which implies that the EFT is not valid. For the heavy $Z^{\prime}$ case, on the other hand, the couplings extend beyond the perturbative regime. 
In the rest of the paper, we only consider the simplified model for the LHC phenomenology, while we mention the EFT approach in the relic density computation and in indirect DM experiments.

\section{Signatures}

In this section we study the signatures of the top flavour-changing DM model in detail. First, we discuss the monotop signal for the LHC Run-II. Then, we consider the limits from the non-collider DM experiments. Lastly, we combine the constraints from the collider and non-collider experiments to determine the viable parameter space of the model.

In the following analyses we follow the strategy described in ref. [61] for new physics simulations. We have implemented the effective Lagrangian (2.2) (as well as the EFT Lagrangian (2.11)) in FeYNRULES2 [62] to create the model files interfaced [63, 64] with MADGraph5_AMC@NLO [65] for the collider study as well as with MicroOMEGAs [66, 67] and MADDM $[68,69]$ for the non-collider study.

\subsection{Monotop at the LHC}

As mentioned in section 1, the top-up flavour-changing DM interaction has been studied in the monotop signature $[3,4,9,27-30]$ and searched for with the CMS detector for the hadronic top decays [26] and with the ATLAS detector for the leptonic top decays [25].

Let us first estimate the constraints on the top-charm flavour-changing DM interaction from the ATLAS-8TeV analysis [25]. The exotic $v_{\text {met }}$ boson in the "non-resonant" model in [25] corresponds to the mediator $Z^{\prime}$ in our model. They assume that only the top-up coupling is non-zero and the $v_{\text {met }}$ boson decays into invisible particles with $100 \%$ branching ratio. They put a bound of about $0.2 \mathrm{pb}$ on the cross section times the leptonic top-decay branching ratio, $\sigma\left(p p \rightarrow t v_{\text {met }}\right) \times B(t \rightarrow b \ell \nu)$, which is approximately independent on the

$v_{\text {met }}$ mass for $m_{v_{\text {met }}}>400 \mathrm{GeV}$. This translates in an upper bound on the $p p \rightarrow t v_{\text {met }}$ production cross section of about $1 \mathrm{pb}$. Although we take into account the visible $Z^{\prime}$ decay, we choose the coupling in eq. (2.9) so that the invisible decay is dominant, and hence we directly apply the upper limit cross section of $1 \mathrm{pb}$ for the $p p \rightarrow t \chi \bar{\chi}$ cross section in our model. Figure 4(left) indicates that the top-up DM model is bounded to have $m_{Z^{\prime}} \gtrsim 800 \mathrm{GeV}$ while the top-charm model is $m_{Z^{\prime}} \gtrsim 400 \mathrm{GeV}$ for our benchmark couplings. We note that the cross sections can be rescaled by varying the coupling $g_{i 3}$ and do not depend on the DM mass as long as the mediator is produced on-shell.

In the following, we will perform a detailed analysis of the LHC Run-II reach on the monotop signature for the top-charm flavour-changing DM model. For the detailed illustration, we take two benchmark points which are characterized by the light or heavy mediator case:

$$
\text { A) } m_{Z^{\prime}}=400 \mathrm{GeV}, \quad \text { B) } m_{Z^{\prime}}=800 \mathrm{GeV} \text {, }
$$

with $m_{\chi}=30 \mathrm{GeV}$ and $\left(g_{\chi}, g_{23}\right)=(3,0.6)$, and present the kinematical distributions to discuss the selection cuts. We then summarise the signal significance on the $\left(m_{\chi}, m_{Z^{\prime}}\right)$ plane for a given value of the couplings. 
At the end of this subsection we propose two strategies to distinguish the top-charm model from the top-up one on the LHC based analyses. The first one exploits the charge asymmetry of the lepton in the final state. The second one makes use of a charm-tagging technique to distinguish charm-quark jets from light-quark $(u, d, s)$ or gluon jets.

\subsubsection{Prospects for the LHC Run-II}

In this section we study in detail the prospects for discovery of the top-charm flavourchanging DM model in proton-proton collisions at a centre-of-mass energy of $13 \mathrm{TeV}$.

Signal. We consider the monotop process (2.10) and also take into account extra jets in the final state. In this paper we focus on the leptonic top decay, $t \rightarrow b+W\left(\rightarrow \ell+\nu_{\ell}\right)$, where $\ell$ is an electron or a muon. Therefore, the signal is characterised by an isolated lepton, a $b$-tagged jet and extra jets in association with large missing energy.

SM background. The following SM backgrounds may mimic the new physics signature:

- Top pair: The semileptonic decays give rise to the similar final state to the signal, and this is the main background after the selection cuts as shown below. The larger jet multiplicity is expected due to the hadronic decay of one of the top quark.

- Single top: Single top production is the only irreducible background. Unless it is produced in association with a $W$ boson we expect the missing energy to be aligned with the lepton since it originates from the same decaying $W$ boson, and hence suitable kinematic cuts can reduce this background efficiently.

- $W+$ jets: The production of a $W$ boson (with a leptonic decay) in association with jets should also be considered since the total cross section is many orders of magnitude larger than the signal.

The presence of only one lepton in the final state for the signal removes all processes with $Z$ bosons from the list of relevant backgrounds. Furthermore the presence of large missing transverse energy, the jet multiplicity, and specific angular distributions of the final-state particles can be exploited to distinguish the signal from the background. These specific features are the motivation behind the cuts that will now be discussed.

We generate the inclusive signal and SM background samples by employing the ME+PS merging scheme with Pythia6 [70], implemented in MadGraph5_AMC@NLO [71]. The fast detector simulation is performed by the DeLPHES3 package [72] with the CMS-based detector setup. We employ MAdAnalysis5 $[73,74]$ for the analyses. The $t \bar{t}$ and single- $t$ cross sections are normalised to $831 \mathrm{pb}$ and $299 \mathrm{pb}$, respectively [75], while the $W+$ jets sample is normalised to $\sigma^{\mathrm{NLO}}\left(W^{ \pm} j\right)$ of about $3 \times 10^{4} \mathrm{pb}[65]$.

Event selection. The final state contains leptons (muons or electrons) and jets as visible objects. Leptons are required to be isolated. ${ }^{2}$ Jets are reconstructed by employing the anti$k_{T}$ algorithm [76] with a radius parameter of 0.5. Leptons and jets are required to have $p_{T}>30 \mathrm{GeV}$ and $|\eta|<2.4$.

\footnotetext{
${ }^{2}$ All the energy surrounding the lepton in the cone $(\Delta R=0.4)$ divided by the lepton $p_{T}$ is below 0.2 .
} 
We pre-select the events by demanding exactly one isolated lepton, $N_{\ell}=1$. In figure 6 we show distributions for the number of jets and some kinematical variables, ${ }^{3}$ which the ATLAS analysis uses, both for the SM background and the signal (benchmarks $A$ and $B$ ) after having required $N_{\ell}=1$.

The following set of cuts are employed in order to maximise the significance of the signal:

$$
\begin{array}{rlrlrl}
N_{\ell} & =1, & 1 & \leq N_{j} \leq 2, & N_{b} & =1, \\
m_{T}\left(\ell, \mathbb{E}_{T}\right) & >m_{T}^{\min }, & \mathbb{E}_{T}>\mathbb{E}_{T}^{\min }, & & |\Delta \phi(\ell, b)|<\Delta \phi^{\max } .
\end{array}
$$

- $1 \leq N_{j} \leq 2$ : The "pure" monotop signal contains exactly one jet which should be $b$-tagged, coming from the top decay, and the most of the previous works including the ATLAS analysis select only the one-jet sample. Here, as discussed, we propose to include an extra jet to enhance the signal and to utilise it to distinguish the topcharm model from the top-up one. The selection is still efficient to reduce the $t \bar{t}$ background in which the average jet multiplicity is higher; see figure 6(top-left). For convenience, we define two signal regions (SRs):

$$
\text { SR1) } N_{j}=1 \text { and } N_{b}=1, \quad \text { SR2) } N_{j}=2 \text { and } N_{b}=1 .
$$

- $N_{b}=1$ : The signal contains one $b$-tagged jet. Requiring exactly one $b$-tagged jet reduces the $W+$ jets background since processes with a $W$ boson in association with bottom quarks are rare, and reduces also the $t \bar{t}$ background where there is a second jet expected to originate from a bottom quark; see figure 6(top-right). For $b$-tagging we use a parametrisation of the efficiency of the combined secondary vertex (CSV) algorithm of the CMS collaboration [77], as a function of the $p_{T}, \eta$ and flavour of the jet. We employ the so-called medium operating point, which overall results in $b$-tagging efficiencies of about $70 \%$ for $b$-flavour jets, about $1 \%$ for $u, d, s$-flavour and gluon jets, and about $20 \%$ for $c$-flavour jets.

- $m_{T}\left(\ell, \mathbb{E}_{T}\right)>m_{T}^{\min }$ : The $m_{T}\left(\ell, \mathbb{E}_{T}\right)$ distributions in figure 6 display a remarkable shape difference between the DM signal and the SM background. For the SM background the $\mathbb{E}_{T}$ and the lepton originates from the same $W$ boson and as a consequence the $m_{T}\left(\ell, \mathbb{E}_{T}\right)$ distribution drops around the $W$ boson mass. This is not the case for the DM signal, where the $\mathbb{E}_{T}$ originates from the invisible $Z^{\prime}$ decay. The heavier $Z^{\prime}$ case (benchmark $B$ ) presents slightly larger $m_{T}$ distribution.

- $\mathbb{E}_{T}>\mathbb{E}_{T}^{\mathrm{min}}$ : The presence of DM in the signal introduces a lot of missing energy in the detector. The missing transverse energy will therefore be much larger on average for the signal in comparison to the SM background processes in which the $\mathbb{E}_{T}$ originates from neutrinos only.

- $|\Delta \phi(\ell, b)|<\Delta \phi^{\max }$ : In the DM signal, the lepton and the $b$-jet always originate from the decay of one top quark and hence they display a small azimuthal angle separation.

\footnotetext{
${ }^{3}$ The transverse mass is defined as $m_{T}\left(\ell, \mathbb{E}_{T}\right)=\sqrt{2 p_{T}^{\ell} \mathbb{E}_{T}\left(1-\cos \Delta \phi\left(\ell, \mathbb{E}_{T}\right)\right)}$.
} 

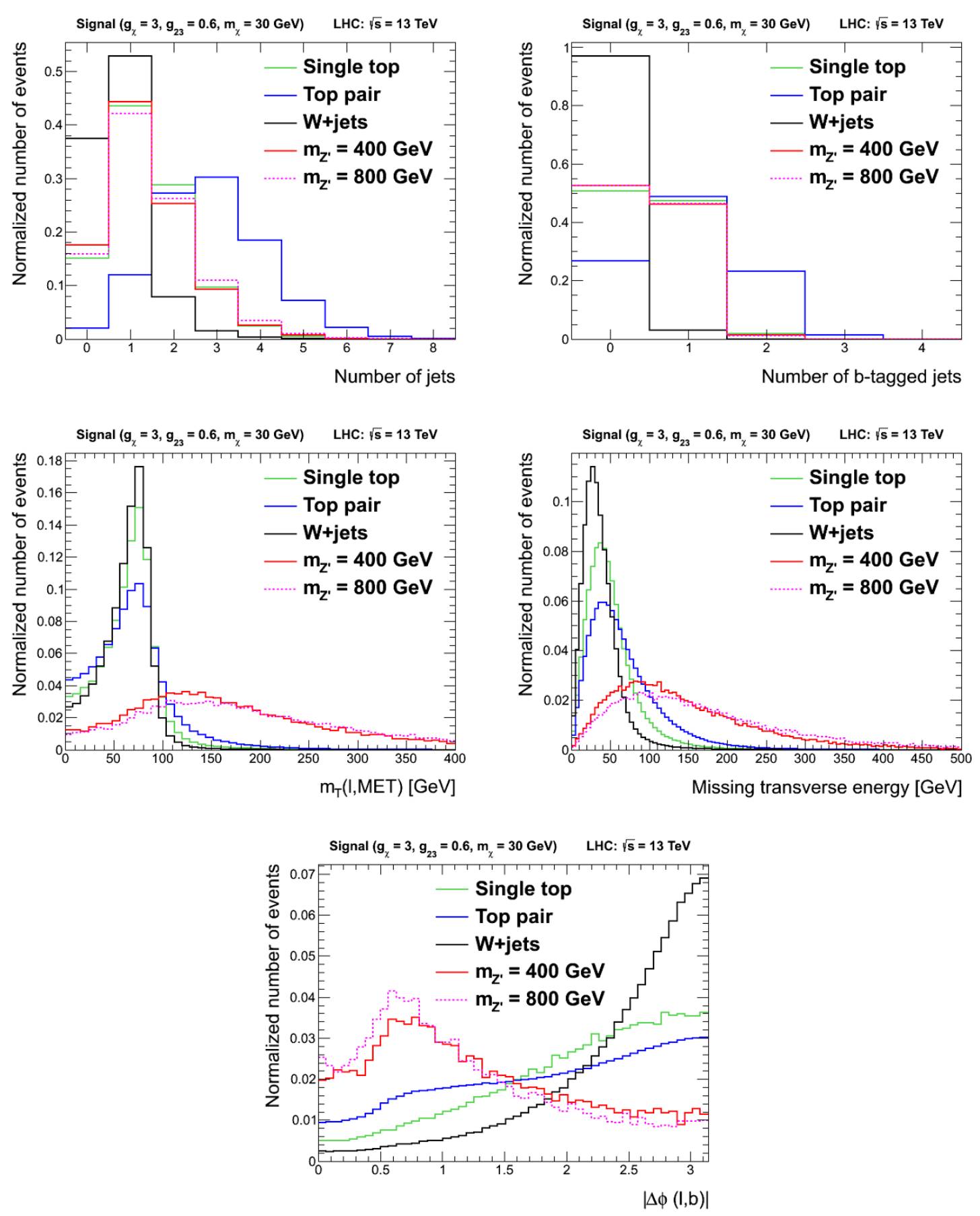

Figure 6. Normalised distributions of the variables used in the analysis for the signal (benchmark point $A$ and $B$ ) and the SM backgrounds after the pre-selection, i.e. we require only $N_{\ell}=1$. For the $\Delta \phi(\ell, b)$ distribution $N_{b}=1$ is also required.

Instead, the SM backgrounds can also present events where the $b$-jet and the lepton arise from different decay chains. In particular, in the $W+$ jets background, the lepton and the $b$-jet are most likely back to back. 


\begin{tabular}{|l||l|l|l|l|l|}
\hline Cuts & Top pair & Single top & $W+$ jets & $A: m_{Z^{\prime} 400}$ & $B: m_{Z^{\prime} 800}$ \\
\hline \hline$N_{\ell}=1$ & $1.56 \times 10^{2}$ & $3.33 \times 10^{1}$ & $3.00 \times 10^{3}$ & $3.74 \times 10^{-1}$ & $2.96 \times 10^{-2}$ \\
\hline$N_{j} \geq 1$ & $1.53 \times 10^{2}$ & $2.83 \times 10^{1}$ & $1.98 \times 10^{3}$ & $3.08 \times 10^{-1}$ & $2.49 \times 10^{-2}$ \\
\hline$N_{j} \leq 2$ & $6.01 \times 10^{1}$ & $2.41 \times 10^{1}$ & $1.93 \times 10^{3}$ & $2.60 \times 10^{-1}$ & $2.02 \times 10^{-2}$ \\
\hline$N_{b}=1$ & $3.15 \times 10^{1}$ & $1.33 \times 10^{1}$ & $8.71 \times 10^{1}$ & $1.45 \times 10^{-1}$ & $1.10 \times 10^{-2}$ \\
\hline
\end{tabular}

Table 2. Cutflow table showing the visible cross sections in pb for the SM backgrounds and the signal for benchmark point $A$ and $B$, consecutively applying the cuts outlined in the text.

Results and discovery reach. We now present the results of our analysis and the discovery reach of the LHC-13TeV with integrated luminosity $\mathcal{L}=100 \mathrm{fb}^{-1}$.

Table 2 shows the visible cross sections for the SM backgrounds and the signal for benchmark point $A$ and $B$ for each consecutive cut up to the $N_{b}=1$ selection. Although the $t \bar{t}$ and single $t$ backgrounds reduce by a factor of ten and the $W+$ jets drops by a factor of a hundred, the background is still larger than the signal.

We now use the information of the kinematical distributions shown in figure 6 , and maximise the statistical signal significance

$$
\mathcal{S} \equiv \frac{S}{\sqrt{S+B}}
$$

to find each optimal cut in the rest of the selection steps. We find that

$$
m_{T}^{\min }=150 \mathrm{GeV}, \quad \notin_{T}^{\min }=200 \mathrm{GeV}, \quad \Delta \phi^{\max }=1.6 .
$$

Table 3 presents the continuation of table 2 together with the signal significance $\mathcal{S}$ assuming the integrated luminosity $\mathcal{L}=100 \mathrm{fb}^{-1}$. The sensitivities of each of the two signal regions (3.3) are also shown at the bottom of the table. The main SM background after all the cuts is $t \bar{t}$. We can easily obtain the significance larger than 5 for the light mediator case (benchmark $A$ ) with $100 \mathrm{fb}^{-1}$. The heavier case (benchmark $B$ ) is instead at reach to be excluded. It is important to note that the signal significance is larger than the pure monotop sample (SR1) when we include an extra jet in the analysis, i.e. SR2. We also note that the shape of the distributions slightly depends on the value of the $Z^{\prime}$ mass. In particular the $m_{T}$ and $\mathbb{E}_{T}$ distributions for heavier $Z^{\prime}$ are centred around larger values. Hence the efficiency of these cuts for the benchmark point $B$ is slightly higher.

The statistical significance $\mathcal{S}$ is not the only representative for an analysis which has to cope with systematic uncertainties, such as the ones on the cross sections of the SM background processes. For instance, given that the $t \bar{t}$ is the most relevant SM background, a large systematic uncertainty will originate from the uncertainty on the $t \bar{t}$ cross section, which was estimated of about $7 \%$ at $\sqrt{s}=8 \mathrm{TeV}$ [78]. Considering also other sources of systematic uncertainties, we conservatively assume a $10 \%$ uncertainty in the SM background estimation and define the significance as

$$
\mathcal{S}^{\prime}=\frac{S}{\sqrt{S+B+(0.1 B)^{2}}}
$$




\begin{tabular}{|l||l|l|l|ll|ll|}
\hline Cuts & Top pair & Single top & $W+$ jets & $A: m_{Z^{\prime} 400}$ & $(\mathcal{S})$ & $B: m_{Z^{\prime} 800}$ & $(\mathcal{S})$ \\
\hline \hline$N_{b}=1$ & $3.15 \times 10^{1}$ & $1.33 \times 10^{1}$ & $8.71 \times 10^{1}$ & $1.45 \times 10^{-1}$ & $(3.98)$ & $1.10 \times 10^{-2}$ & $(0.30)$ \\
\hline$m_{T}>150 \mathrm{GeV}$ & $1.86 \times 10^{0}$ & $1.74 \times 10^{-1}$ & $3.25 \times 10^{-1}$ & $7.83 \times 10^{-2}$ & $(15.86)$ & $6.76 \times 10^{-3}$ & $(1.39)$ \\
\hline $\mathbb{E}_{T}>200 \mathrm{GeV}$ & $1.19 \times 10^{-1}$ & $6.48 \times 10^{-3}$ & $5.17 \times 10^{-3}$ & $3.21 \times 10^{-2}$ & $(25.18)$ & $3.38 \times 10^{-3}$ & $(2.92)$ \\
\hline$|\Delta \phi|<1.6$ & $8.61 \times 10^{-2}$ & $4.97 \times 10^{-3}$ & $2.27 \times 10^{-3}$ & $3.08 \times 10^{-2}$ & $(27.62)$ & $3.24 \times 10^{-3}$ & $(3.30)$ \\
\hline \hline SR1: $N_{j}=1$ & $3.76 \times 10^{-2}$ & $2.30 \times 10^{-3}$ & $1.29 \times 10^{-3}$ & $1.77 \times 10^{-2}$ & $(23.10)$ & $1.82 \times 10^{-3}$ & $(2.77)$ \\
\hline SR2: $N_{j}=2$ & $4.86 \times 10^{-2}$ & $2.67 \times 10^{-3}$ & $9.82 \times 10^{-4}$ & $1.30 \times 10^{-2}$ & $(16.15)$ & $1.43 \times 10^{-3}$ & $(1.95)$ \\
\hline
\end{tabular}

Table 3. Cutflow table showing the visible cross sections in pb for the SM backgrounds and the signal for benchmark point $A$ and $B$, after the $N_{b}=1$ selection. The columns $\mathcal{S}$ show the statistical signal significance $(3.4)$ for $\mathcal{L}=100 \mathrm{fb}^{-1}$.

\begin{tabular}{|l||l|l|l|lr|lr|}
\hline Cuts & Top pair & Single top & $W+$ jets & $A: m_{Z^{\prime} 400}$ & $\left(\mathcal{S}^{\prime}\right)$ & $B: m_{Z^{\prime} 800}$ & $\left(\mathcal{S}^{\prime}\right)$ \\
\hline \hline$N_{b}=1$ & $3.15 \times 10^{1}$ & $1.33 \times 10^{1}$ & $8.71 \times 10^{1}$ & $1.45 \times 10^{-1}$ & $(0.01)$ & $1.10 \times 10^{-2}$ & $(0.00)$ \\
\hline$m_{T}>300 \mathrm{GeV}$ & $1.09 \times 10^{-1}$ & $1.24 \times 10^{-2}$ & $1.19 \times 10^{-2}$ & $2.15 \times 10^{-2}$ & $(1.61)$ & $2.43 \times 10^{-3}$ & $(0.18)$ \\
\hline$E_{T}>350 \mathrm{GeV}$ & $3.38 \times 10^{-3}$ & $1.74 \times 10^{-4}$ & $5.84 \times 10^{-5}$ & $4.04 \times 10^{-3}$ & $(8.89)$ & $6.69 \times 10^{-4}$ & $(1.61)$ \\
\hline$|\Delta \phi|<1.0$ & $1.87 \times 10^{-3}$ & $1.28 \times 10^{-4}$ & $0.00 \times 10^{0}$ & $3.76 \times 10^{-3}$ & $(12.03)$ & $6.07 \times 10^{-4}$ & $(2.36)$ \\
\hline \hline SR1: $N_{j}=1$ & $6.42 \times 10^{-4}$ & $6.41 \times 10^{-5}$ & $0.00 \times 10^{0}$ & $1.75 \times 10^{-3}$ & $(10.09)$ & $2.76 \times 10^{-4}$ & $(2.27)$ \\
\hline SR2: $N_{j}=2$ & $1.23 \times 10^{-3}$ & $6.41 \times 10^{-5}$ & $0.00 \times 10^{0}$ & $2.00 \times 10^{-3}$ & $(8.99)$ & $3.31 \times 10^{-4}$ & $(1.82)$ \\
\hline
\end{tabular}

Table 4. Same as table 3 , but with the tighter cuts. The columns labelled $\mathcal{S}^{\prime}$ show the signal significance including a $10 \%$ systematic uncertainty (3.6) for $\mathcal{L}=100 \mathrm{fb}^{-1}$.

We repeat the same procedure, but maximise the significance (3.6) to find a new set of optimal cuts. We find a tighter set of cuts

$$
m_{T}^{\min }=300 \mathrm{GeV}, \quad \mathbb{E}_{T}^{\min }=350 \mathrm{GeV}, \quad \Delta \phi^{\max }=1.0 .
$$

Table 4 gives the results of these selection cuts, and clearly shows that even after including systematic uncertainties the potential remains to discover the top-charm flavour-changing DM events during the run of the LHC with $13 \mathrm{TeV}$ proton-proton collisions and an expected $100 \mathrm{fb}^{-1}$ of collected data.

In order to establish the reach of the LHC in the parameter space of the model, we perform a parameter scan in the $m_{\chi}-m_{Z^{\prime}}$ plane. Figure 7 shows the three sigma and five sigma contours of the signal significance without $(\mathcal{S})$ and with $\left(\mathcal{S}^{\prime}\right)$ a systematic uncertainty in this mass plane. There is essentially no dependence on the DM mass since the mediator is always produced on-shell in this mass range, and subsequently decaying into a pair of the DM particles. On the other hand, the LHC reach is largely dependent on the mediator mass that determines the production cross section for a given coupling. We find that a large part of the mass space is accessible in the $13 \mathrm{TeV}$ run of the LHC for the reasonable choice of the coupling parameter such as $\left(g_{\chi}, g_{23}\right)=(3,0.6)$.

Following the same analysis strategy, one can easily explore the corresponding top-up flavour-changing DM model, setting $g_{\chi}=3.0$ and $g_{13}=0.6$ and varying the DM and the $Z^{\prime}$ masses. The enhancement of the production cross section due to the up-quark PDF (see figure 4) determines a much higher reach for the top-up model with respect to the 


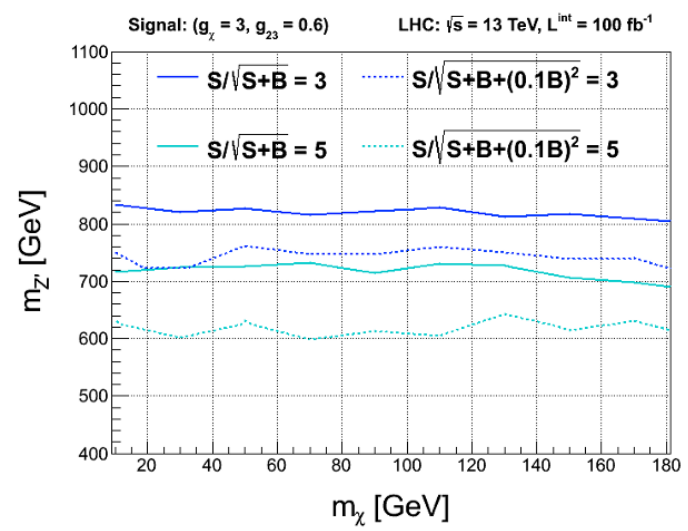

Figure 7. 3 and $5 \sigma$ contours of the signal significance in the $m_{\chi}-m_{Z^{\prime}}$ parameter space without (solid) and with (dotted) including a systematic uncertainty in the calculation of the significance.

top-charm model for analogous values of the couplings. Indeed we find that the top-up DM model can be discovered at the LHC-13TeV for a $Z^{\prime}$ mass up to about $1.5 \mathrm{TeV}$.

\subsubsection{Top-charm vs. top-up in monotop}

As discussed, the top-charm and top-up flavour-changing DM models essentially give the same monotop signature at the LHC. The main difference is the overall cross sections, and hence the mass reach is different if we assume the same couplings between the two models. However, there is no other direct observable which is related to the $Z^{\prime}$ mass, since we have seen in figure 6 that the kinematical distributions are similar between the different $Z^{\prime}$ mass. Hence, even if the monotop signal is discovered at the LHC Run-II, it may be very difficult to discriminate between the top-charm and the top-up DM models.

In this subsection we propose possible techniques to distinguish between the two models in the monotop signature, not based on the overall signal cross sections. For this purpose we define two benchmarks for the top-up DM model:

$$
\begin{aligned}
& \text { A) } \quad\left\{g_{\chi}, g_{13}, m_{\chi}, m_{Z^{\prime}}\right\}=\{3.0,0.19,30 \mathrm{GeV}, 400 \mathrm{GeV}\} \\
& \text { B) }\left\{g_{\chi}, g_{13}, m_{\chi}, m_{Z^{\prime}}\right\}=\{3.0,0.19,30 \mathrm{GeV}, 800 \mathrm{GeV}\}
\end{aligned}
$$

where we choose the same parameters as in the benchmarks of the top-charm model (3.1) except the $g_{13}$ coupling. The value of the coupling $g_{13}$ is chosen so that the top-up monotop cross sections become comparable to the top-charm ones.

Lepton charge asymmetry. The first strategy that we adopt to distinguish the topcharm flavour-changing DM model from the top-up one is to exploit the lepton charge asymmetry in the leptonic monotop final state. Since the up-quark PDF is much larger than the up-antiquark one in protons, the monotop process for the top-up DM model in proton-proton collisions produces much more top quarks than top anti-quarks, leading to a large majority of events with a positively charged lepton [28]. For the top-charm model, on the other hand, we expect an equivalent number of events with a positively and negatively charged lepton as the charm PDF is equal to the charm-antiquark one. 


\begin{tabular}{|l|r|r|r||r|r|r|}
\hline & \multicolumn{3}{|c||}{ Top-charm model } & \multicolumn{3}{c|}{ Top-up model } \\
\cline { 2 - 7 } & $\ell^{+}+\ell^{-}$ & $\ell^{+}$ & $\ell^{-}$ & $\ell^{+}+\ell^{-}$ & $\ell^{+}$ & $\ell^{-}$ \\
\hline $\mathcal{S}\left(A: m_{Z^{\prime}}=400 \mathrm{GeV}\right)$ & 27.62 & 19.75 & 19.30 & 30.19 & 34.81 & 4.78 \\
\hline $\mathcal{S}\left(B: m_{Z^{\prime}}=800 \mathrm{GeV}\right)$ & 3.30 & 2.34 & 2.33 & 4.88 & 6.28 & 0.48 \\
\hline \hline $\mathcal{S}^{\prime}\left(A: m_{Z^{\prime}}=400 \mathrm{GeV}\right)$ & 12.03 & 9.35 & 9.73 & 13.98 & 16.30 & 2.70 \\
\hline $\mathcal{S}^{\prime}\left(B: m_{Z^{\prime}}=800 \mathrm{GeV}\right)$ & 2.36 & 1.85 & 2.17 & 4.05 & 5.55 & 0.47 \\
\hline
\end{tabular}

Table 5. Signal significance for the benchmark point $A$ and $B$ for each positively and negatively charged lepton in the final state.

In order to quantify this observation, we look at the signal significance again, but for positively and negatively charged leptons separately. Note that the main SM background, i.e. $t \bar{t}$, is charge symmetric. In table 5 we report the result of these investigations for both the top-charm (3.1) and the top-up (3.8) benchmarks. We display the significance (both without and with a systematic uncertainty) for the combined signal region SR1+SR2 of each benchmark depending on the lepton charge selection. The $\ell^{+}+\ell^{-}$columns correspond to the analysis discussed in the previous section, which does not distinguish the lepton charge.

The charge selection can efficiently distinguish between the top-charm and top-up DM models. For the top-up model, the significance increases (decreases) remarkably by requiring only positively (negatively) charged leptons. The predominance of positively charged configurations in the top-up model implies that the significance of the analysis targeted to a positively charge lepton is even larger than the one without the charge identification. For the top-charm DM model, on the other hand, the significance for the positively charged case and the negatively charged case are essentially equivalent, and they are both smaller than the combined one. In short,

$$
\begin{array}{ll}
\mathcal{S}_{\ell^{+}+\ell^{-}}>\mathcal{S}_{\ell^{+}} \simeq \mathcal{S}_{\ell^{-}} & \text {for the top-charm DM model }, \\
\mathcal{S}_{\ell^{+}}>\mathcal{S}_{\ell^{+}+\ell^{-}} \gg \mathcal{S}_{\ell^{-}} & \text {for the top-up DM model },
\end{array}
$$

and we conclude that the lepton charge identification provides an efficient technique to distinguish between the top-charm and top-up DM models in the monotop signature.

Charm-jet tagging. The second strategy that we investigate to distinguish between the top-charm and the top-up models makes use of a charm tagging for an extra jet in SR2.

Such a charm-tagging algorithm has been recently released by ATLAS [79] and it exploits the properties of displaced tracks, reconstructed secondary vertices and soft leptons inside jets. In our analysis, we assume a constant tagging efficiency for simplicity, without any dependence on $p_{T}$ and $\eta$ of the jets. This is accurate enough for a first estimation of the effect of charm tagging as the $p_{T}$ and $\eta$ dependence is quite mild [79]. Based on the needs of a specific analysis, different working points can be chosen to select the desired charm-jet tagging efficiency and to either improve the rejection of light-flavour jets or bottom-quark jets.

To select the charm-flavour jet with a low mistag rate for light $(u, d, s, g)$ jets, we employ a tight $c$-tagging working point. Taking inspiration from the efficiency performance 


\begin{tabular}{|l|r|r|r||r|r|r|}
\hline & \multicolumn{3}{|c||}{ Before $c$-tagging } & \multicolumn{3}{c|}{ After $c$-tagging } \\
\cline { 2 - 7 } & SR1 & SR2 & SR1+2 & SR1 & SR2 & SR1+2 \\
\hline $\mathcal{S}($ top-up; $A)$ & 24.78 & 18.10 & 30.19 & 24.78 & 1.19 & 24.44 \\
\hline $\mathcal{S}($ top-charm; $A)$ & 23.10 & 16.15 & 27.62 & 23.10 & 2.30 & 23.02 \\
\hline \hline $\mathcal{S}^{\prime}($ top-up; $A)$ & 12.64 & 9.46 & 13.98 & 12.64 & 1.52 & 12.72 \\
\hline $\mathcal{S}^{\prime}($ top-charm; $A$ ) & 10.19 & 8.99 & 12.03 & 10.19 & 2.74 & 10.49 \\
\hline
\end{tabular}

Table 6. Signal significance for the benchmark point $A$ in both top-up and top-charm models before and after the charm-tagging requirement.

reported by the ATLAS Collaboration [79], we assume an overall $c$-tagging efficiency of $20 \%$ for $c$-flavour jets, $1 \%$ for $u, d, s$-flavour and gluon jets, and $15 \%$ for $b$-flavour jets. We note that the mistag rate is extremely low, but we are compromised by the rather low $c$-tagging efficiency. Ideally, if the charm tagger would have a $100 \%$ efficiency, the signal cross section would be suppressed by roughly a factor of a hundred for the top-up model, while by about a factor of three for the top-charm model, as seen in figure 4.

Considering the same cuts of the previous section, we require the second jet of the SR2 to be a charm-tagged jet, and compute the significance for the benchmark point $A\left(m_{Z^{\prime}}=400 \mathrm{GeV}\right)$ for both top-up and top-charm models. Table 6 shows that after $c$-tagging the significance of the SR2 for the top-charm model becomes twice larger than that for the top-up model although the signal significance itself suffers a sharp drop for both models.

Even though this technique is probably much less efficient than the lepton charge asymmetry, it represents nevertheless an alternative strategy to distinguish between the top-up and top-charm DM models in the monotop signature. More detailed investigations of charm-tagging techniques could result in better performances in the search for DM described in this paper.

\subsection{Canonical dark matter searches}

A stable new particle that interacts predominantly with a top-charm or a top-up quark pair has interesting implications for DM phenomenology, in particular for relic density and canonical DM searches such as direct and indirect detection experiments. In the following we discuss in detail these considerations, devoting special attention to a tentative explanation of the galactic centre excess in terms of flavour-changing DM.

Relic density. As mentioned in section 2, we focus on the parameter space where $m_{Z^{\prime}}$ is parametrically the highest scale of the theory. In this case, the typical energy scale of the thermal freeze-out process is smaller than the mass of the mediator $m_{Z^{\prime}}$. We then expect that the description of the dynamics in terms of a simplified model or an EFT makes no practical difference. $^{4}$ Indeed, for the parameter points of interest, we checked that the results in the simplified model (2.2) and in the EFT description (2.11) agree very well, by using MicroOMEGAs [66, 67] and MAdDM [68, 69].

\footnotetext{
${ }^{4}$ Apart from parametrically separating $m_{Z^{\prime}}$ from the other scales of the theory, one has to ensure that the width of $Z^{\prime}$ is not too large, i.e. $\Gamma_{Z^{\prime}}<m_{Z^{\prime}}$.
} 


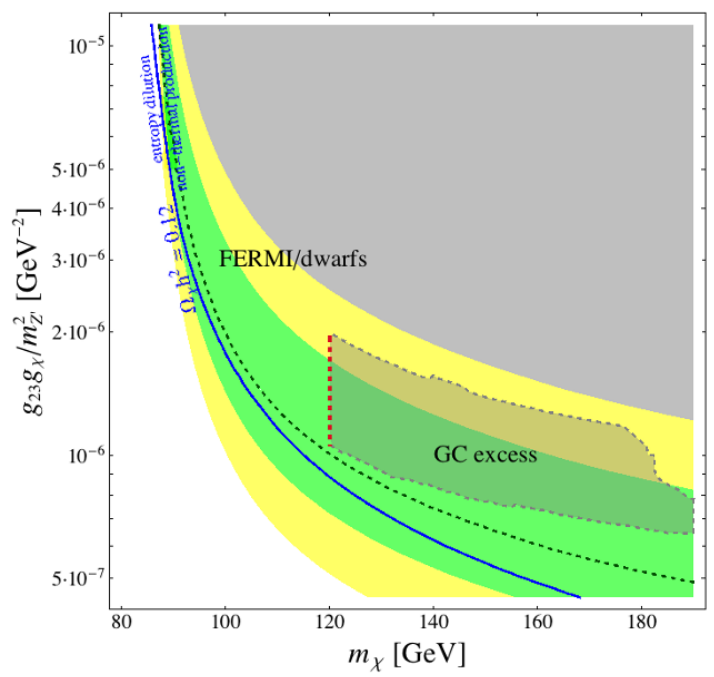

(a) We assume the observed DM relic density in all of the parameter plane. The area within the dashed boundary shows the parameter region that can fit the galactic centre excess. The grey area is excluded by FERMI.

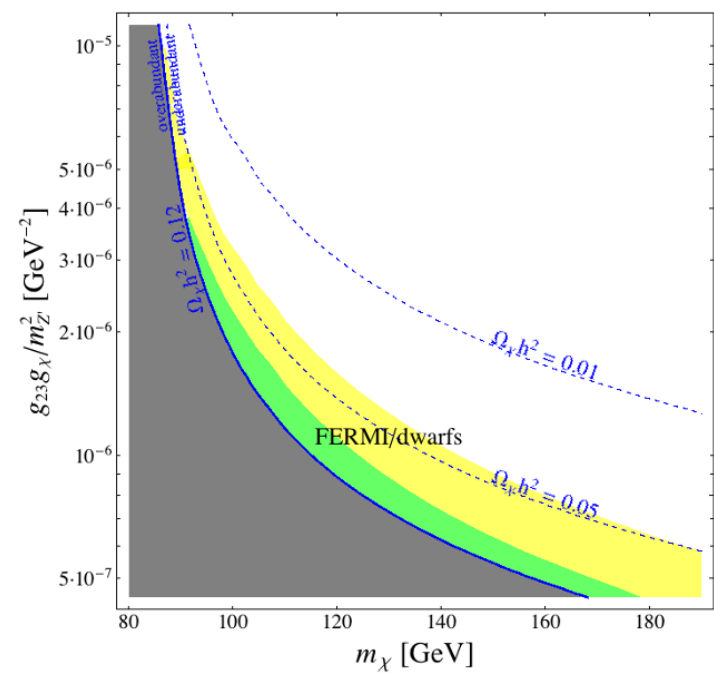

(b) We do not make such assumption as in figure 8(a), and hence the FERMI limit is rescaled according to the relic density of the model. The dark grey area is excluded by the DM overabundance.

Figure 8. Relic density contours (blue lines), limits from FERMI (dashed green line with green $(1 \sigma)$ and yellow $(2 \sigma)$ expected uncertainty bands), and the parameter region that fits the galactic centre excess (light grey region) for the top-charm flavour-changing DM model.

In the simplified model, the annihilation of the DM candidate to the quark pair during the thermal freeze-out occurs exclusively via $s$-channel mediation of a $Z^{\prime}$ boson, as shown in eq. (2.4). The relic density of $\chi$ can be equal to the observed relic density of DM, $\Omega_{\chi} h^{2}=0.12$ [80] for reasonable values of the effective coefficient $g_{i 3} g_{\chi} / m_{Z^{\prime}}^{2}$. This is illustrated for the top-charm model in figure 8, while the corresponding plot for the top-up model looks practically the same, as the mass difference between the charm and up quarks has little effect on both the calculation of the relic density and the photon fluxes of the annihilation products.

In figure $8(\mathrm{a})$, the proper relic density is depicted by the blue contour, however it is assumed that the relic density of $\chi$ is equal to the observed DM relic density in all of the parameter space. For $m_{\chi}<m_{t} / 2$ the annihilation to $t-c$ or $t-u$ quark pair is kinematically forbidden and the correct relic density is achieved if we invoke extra dynamics, e.g. an entropy dilution mechanism. For values of the parameter space to the right of the $\Omega_{\chi} h^{2}=0.12$ line, $\chi$ would annihilate too much and the proper relic density is achieved if we assume non-thermal production. In figure $8(\mathrm{~b})$, on the other hand, we do not make such assumptions, so that the region of the parameter space with overabundant DM is excluded while in the region where DM is underabundant we provide indicative relic density contours.

Indirect searches. The latest FERMI data on photon fluxes from dwarf spheroidal galaxies of the Milky Way [81] provide strong constraints on the parameter space of the model, as illustrated in figure 8. Although the published results do not include annihilation 
to flavour-violating pairs such as $t-c / u$, the photon flux is very similar to that of $b \bar{b}$ for the DM mass range under focus [82]. Therefore, the limit on DM annihilating to $t-c / u$ is similar to that of a bottom-quark pair. The dashed green line shows the observed limit while the green and yellow bands depict the $1 \sigma$ and $2 \sigma$ uncertainties of the expected sensitivity. We notice that the observed limit is nearly probing the thermal relic density line of the model. In figure $8(\mathrm{~b})$ we show the FERMI limits rescaled by $\left(\Omega_{\chi} h^{2} / 0.12\right)^{2}$ to account for the varying relic density. We note that, while in figure 8(a) FERMI excludes all the large $m_{\chi}$ region (because of the increased annihilation), in figure 8(b) the decreased relic density dominates over the increased cross section so that FERMI turns out to be sensitive only up to the $\Omega_{\chi} h^{2} \simeq 0.05$ line.

The latest results from the searches for antiprotons by the AMS-02 experiment [83] can also be used to set limits on DM annihilation in the centre of our galaxy. The reach of these limits depends on the uncertainties of the astrophysical background and the propagation of the antiprotons in the galaxy [84-86], and under reasonable assumptions the limit on DM annihilating to $b \bar{b}$ is equivalent or even stronger than the one obtained from FERMI. Due to the relatively larger uncertainties, we have not used these limits in this work, however, it would be interesting to see how much further they can constrain the models discussed here.

We also report on the excess of gamma rays from the galactic centre that has been observed [34] and updated [35] by the FERMI telescope. Initial proposals that fit the photon profile of the excess included DM annihilating to $b \bar{b}$ pairs (see eg $[87,88]$ for phenomenological analyses). The top-charm model leads to a simlar photon flux, and hence can fit the excess [33]. In figure 8(a) we show, based on the results of [33], the region of the parameter space of the model that can fit the galactic centre excess. The fit to the excess corresponds to DM that is slightly over-annihilating, so that a non-thermal production mechanism is required to ensure the observed relic density. The abrupt stop at $m_{\chi}=120 \mathrm{GeV}$ is an artifact of extracting photon fluxes from PPPC [82]; in principle one expects the fit region to expand to lower DM masses. ${ }^{5}$ We also note that, since the photon flux from an up quark is practically the same to that from a charm quark [82], the top-up model fits the galactic centre excess, too.

Direct searches. The DM candidate $\chi$ in the top flavour-changing model has radiatively induced flavour-conserving interactions with quarks so that, in principle, direct search experiments can be relevant. ${ }^{6}$

The reactor response depends on the strength of the interaction between the DM candidate and the reactor nucleus, typically described in terms of non-relativistic effective operators that in general depend on the momentum exchange between the two particles, their relative velocity and their spins. These, in turn, are described in terms of effective $\chi-N$ operators, themselves described in terms of a set of effective interactions between $\chi$ and the quarks or gluons.

\footnotetext{
${ }^{5}$ The photon flux for annihilation to a top-quark pair given in PPPC starts from $m_{\chi}=180 \mathrm{GeV}$. When recasting the top-pair and charm-pair fluxes to get the flux of the top-charm pair, this translates into a minimum value of $m_{\chi}=120 \mathrm{GeV}$.

${ }^{6}$ There are also effective interactions with gluons which are however higher dimensional and two-loops suppressed, therefore they are negligible in our simplified model.
} 
In describing the scattering of the DM candidate against nuclei for low-energy experiments, we can take the limit of small relative velocity and momentum transfer. In this limit and neglecting higher derivative operators, the scalar $(\bar{\chi} \chi \bar{q} q)$ and vector $\left(\bar{\chi} \gamma^{\mu} \chi \bar{q} \gamma_{\mu} q\right)$ DMquark interactions contribute to the scattering cross section that does not depend on the spin of the colliding particles, the axial-vector $\left(\bar{\chi} \gamma^{\mu} \gamma^{5} \chi \bar{q} \gamma_{\mu} \gamma^{5} q\right)$ and tensor $\left(\bar{\chi} \sigma^{\mu \nu} \chi \bar{q} \sigma_{\mu \nu} q\right)$ interactions contribute to spin-dependent scattering, while all the other effective operators can be neglected.

For the top-up model the interaction of DM with a nucleus is achieved via box diagrams with two $Z^{\prime}$ bosons, one top quark and one DM field running in the loop, while for the top-charm model the connection with the valence quarks of the nucleon requires a second loop. We have calculated them in the zero momentum transfer limit for the top-up model and matched the Wilson coefficients to the simplified model computation. Regarding the spin-independent cross sections, in the limit of a massless up-quark, the contribution to the scalar operator vanishes, while the finite contribution to the vector operator $c_{V} \bar{\chi} \gamma^{\mu} \chi \bar{q} \gamma_{\mu} q$ is given by the following Wilson coefficient:

$c_{V}=6 g_{\chi}^{2} g_{13}^{2} \int_{0}^{1} d x \int_{0}^{1-x} d y x\left[\frac{3 m_{\chi}^{2}}{2 m_{Z^{\prime}}^{2}} z\left(I_{l_{1}^{2}}^{n=4}-I_{l_{2}^{2}}^{n=4}\right)-m_{\chi}^{2} z\left(I_{0_{1}}^{n=4}-I_{0_{2}}^{n=4}\right)+2 \int_{0}^{1-x-y} d z z m_{\chi}^{2}\left(I_{l_{1}^{2}}^{n=5}-I_{l_{2}^{2}}^{n=5}\right)\right]$,

where $z=1-x-y$ when it is not the variable of integration and

$$
\begin{aligned}
& I_{0_{i}}^{n}=\int \frac{d^{4} l_{i}}{(2 \pi)^{4}} \frac{1}{\left(l_{i}^{2}-\Delta_{i}\right)^{n}}=2 i \frac{(-1)^{n}}{16 \pi^{2}} \frac{\Gamma(n-2)}{\Gamma(n)} \frac{1}{\Delta_{i}^{n-2}}, \\
& I_{l_{i}^{2}}^{n}=\int \frac{d^{4} l_{i}}{(2 \pi)^{4}} \frac{l_{i}^{2}}{\left(l_{i}^{2}-\Delta_{i}\right)^{n}}=i \frac{(-1)^{n-1}}{16 \pi^{2}} \frac{\Gamma(n-3)}{\Gamma(n)} \frac{1}{\Delta_{i}^{n-3}},
\end{aligned}
$$

with

$$
\begin{aligned}
& l_{1}=q+y k-z p, \quad \Delta_{1}=(y k-z p)^{2}+x m_{Z^{\prime}}^{2}-y\left(m_{u}^{2}-m_{t}^{2}\right), \\
& l_{2}=q+y k+z p, \quad \Delta_{2}=(y k+z p)^{2}+x m_{Z^{\prime}}^{2}-y\left(m_{u}^{2}-m_{t}^{2}\right),
\end{aligned}
$$

where $p, k$ and $q$ are the DM, the quark and the loop momentum respectively. For reasonable values of the model parameters, the size of the Wilson coefficient turns out to be $\lesssim 10^{-50} \mathrm{~cm}^{2}$, orders of magnitude smaller than current observational sensitivities, due to the cancellation among the box diagrams. This is true also for the spin-dependent cross section, where the experimental constraints are less strong. Therefore, the top-up DM model is not constrained by direct search experiments and consequently, neither is the top-charm model.

\subsection{Complementarity between the LHC and non-collider experiments}

In the previous subsections we have studied the LHC and non-collider phenomenology separately for the top flavour-changing simplified DM model. Here we combine the two analyses to provide a complete picture of the experimental reach on the parameter space of the model and the complementarity among different DM search experiments. 

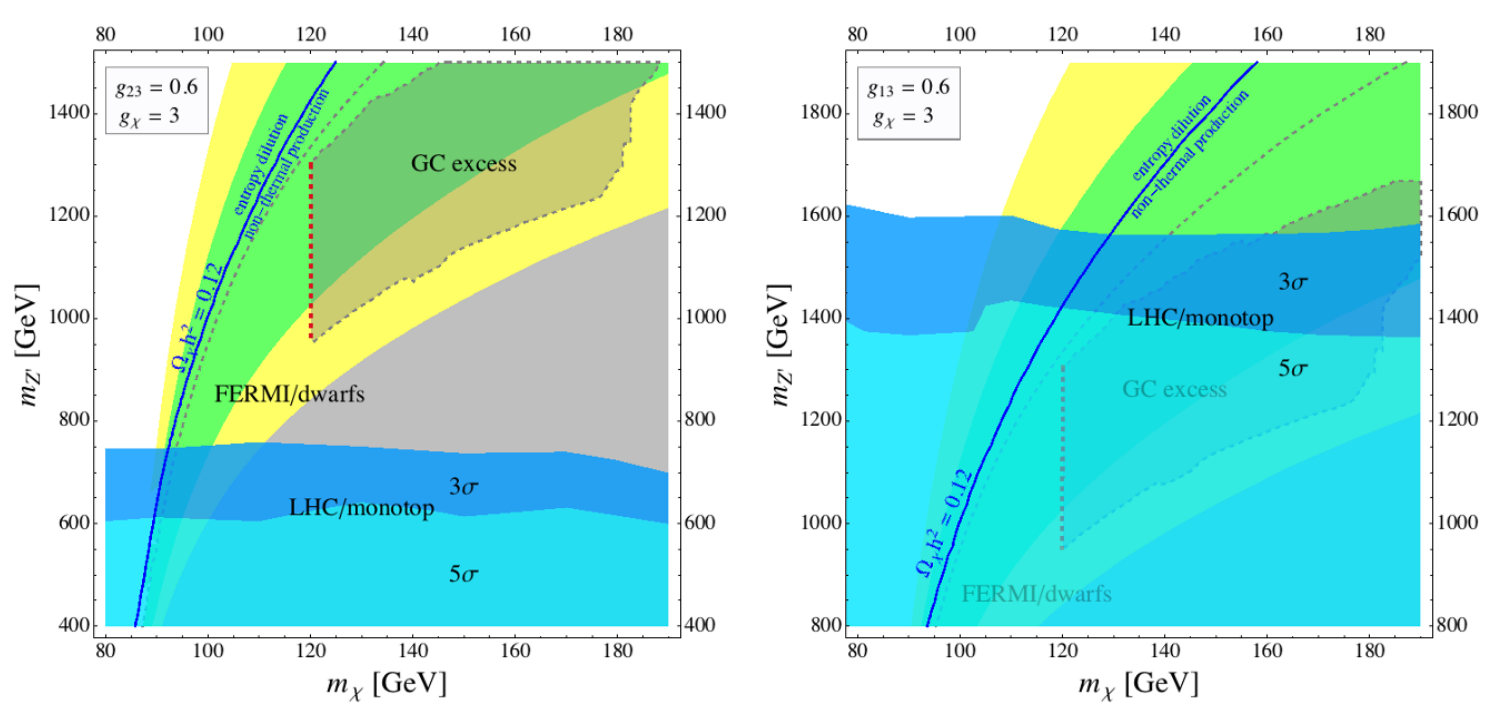

Figure 9. Aggregated figures of the relic density, FERMI limits and LHC reach for the DM candidate of the top-charm (left) and top-up (right) flavour-changing models in the DM-mediator mass plane, where we assume the observed DM relic density in all of the parameter plane. The grey area with dashed boundary shows the parameter region that can fit the galactic centre excess. The dashed green line is the observed FERMI limit, while the green and yellow bands correspond to $1 \sigma$ and $2 \sigma$ uncertainties on the expected limit. The dark and light blue bands depict the $3 \sigma$ and $5 \sigma$ reach of monotop searches at LHC- $13 \mathrm{TeV}$ with $100 \mathrm{fb}^{-1}$.

The results are summarised in figure 9, where we show the prospects from LHC-13TeV and the FERMI constraints together with the region of the parameter space that fits the tentative galactic centre excess for both the top-charm and the top-up models. In these plots the FERMI limits are obtained assuming that the relic density of the DM is equal to the observed one in all the parameter plane, allowing for other mechanisms than thermal production, same as in figure 8(a). If we would instead assume only thermal production for the DM candidate, the observed relic abundance is obtained only along the blue lines and on the rest of the parameter space the bounds from FERMI are much weaker (see figure 8(b) and discussion there). The LHC-13TeV reach, on the other hand, does not depend on these assumptions. This is already a basic difference between the limits derived from colliders and from indirect detection.

The LHC reach depends almost exclusively on the mediator mass, which sets the size of the cross section (for fixed couplings). On the other hand, the reach of indirect detection experiments depends also on the DM mass, which affects the efficiency of the DM annihilation. This implies that the LHC and indirect DM experiments can probe different regions of the parameter space of the model.

Another interesting point we observe is that, by analysing the two plots in figure 9, a combined interpretation of the top flavour-changing DM models at LHC-13TeV and in indirect DM searches reveals different features between the top-charm and the top-up DM model. 
In the top-charm DM model (figure 9(left)), the FERMI exclusion covers most of the parameter space that can be probed by LHC-13TeV. However, the blue line where the dark matter abundance is obtained via usual thermal production is not constrained by FERMI, and instead it will be probed by LHC- $13 \mathrm{TeV}$ for a DM mass around $90 \mathrm{GeV}$. The region capable of explaining the galactic center excess, characterized by the mediator heavier than a $\mathrm{TeV}$, lies beyond the reach of $\mathrm{LHC}-13 \mathrm{TeV}$.

The top-up DM model (figure 9(right)) presents the same limit from indirect detection as in the top-charm DM model but has a much larger reach at LHC-13TeV. The LHC$13 \mathrm{TeV}$ will be able to probe the thermal relic DM line up to a mass of around $130 \mathrm{GeV}$, and to cover almost completely the region capable of accommodating the galactic center excess.

Even though in the figures we have fixed the coupling as $\left(g_{\chi}, g_{i 3}\right)=(3,0.6)$, the previous discussion is robust under modifications of the $g_{i 3}$ coupling as long as the invisible decay of the $Z^{\prime}$ remains dominant. This is due to the fact that the monotop signature scales as $g_{i 3}^{2} / m_{Z^{\prime}}^{4} \times B\left(Z^{\prime} \rightarrow \chi \bar{\chi}\right)$ and the DM annihilation scale as $g_{i 3}^{2} g_{\chi}^{2} / m_{Z^{\prime}}^{4}$. Hence, reducing the coupling $g_{i 3}$ (keeping the $Z^{\prime}$ invisible decay as the dominant one) will shift down by the same amount both the region capable of fitting the galactic centre excess as well as the $3 \sigma$ and $5 \sigma$ discovery lines of LHC-13TeV, and thus it will not affect qualitatively our conclusions. In this perspective, one can argue that the monotop signature at the LHC and the canonical DM searches in this simplified model allow for a straightforward comparison, because of their similar scaling with the couplings.

\section{Conclusions and discussions}

In this work we have studied the phenomenology of a simplified model of DM with flavourchanging interactions. Given the strong constraints on flavour-changing interactions of the down-quark sector from low-energy experiments, we focused on DM interacting with a right-handed top-up or top-charm pair via a neutral vector mediator $Z^{\prime}$. The simplified model is parametrised by the mass of the DM candidate, the mass of the mediator and the couplings of the $Z^{\prime}$ to the DM and the quark pair. Depending on these parameters, the model provides rich signatures at colliders as well as at non-collider experiments, as summarised in table 1 and described in section 2.1.

We focused on the top-charm flavour-changing DM model whose most relevant signature at the LHC is a single top quark plus missing energy, i.e. a monotop final state. For our benchmark point $g_{\chi}=3$ and $g_{23}=0.6$, the limit from LHC-8TeV is approximately $m_{Z^{\prime}} \gtrsim 400 \mathrm{GeV}$. For the prospects of LHC-13TeV with $100 \mathrm{fb}^{-1}$, we find that, for the same couplings, the $3 \sigma(5 \sigma)$ reach can go up to $m_{Z^{\prime}} \sim 760(640) \mathrm{GeV}$, roughly independent of the DM mass. We then discussed how to distinguish the top-charm DM model from the top-up one in the monotop signatures by making use of lepton charge determination and by employing a charm-tagging technique.

For non-collider DM signatures, we showed that the DM candidates with top flavourchanging interactions can be thermal relics for reasonable values of couplings and for a mass of the order of the electroweak scale. We found that direct searches do not pose bounds on the simplified models under study, due to the cancellation of the box diagrams 
involved in the scattering of DM against nuclei. On the other hand, indirect searches pose strong bounds. We used the results from FERMI on photon fluxes from dwarf spheroidal galaxies to constrain the parameter space of the models and identified the part of the parameter space that fits the galactic centre excess. Since the photon fluxes of the top-up and top-charm models are practically same, both models fit the excess equally well.

Finally, combining the LHC and non-collider analyses, we showed the complementarity among the different DM search experiments in probing the parameter space of the model and how the combination of these analyses will be able to distinguish between the topcharm and top-up flavour-changing DM models.

Before closing, we would like to comment on the UV completion of the model for what concerns the origin of the flavour-changing couplings and the extra degrees of freedom needed in order to make the model anomaly free. Considerations regarding the UV completion of simplified models with $Z^{\prime}$ bosons have been recently discussed in [89].

One way to build the flavour-changing terms is to impose different charges under the $\mathrm{U}(1)^{\prime}$ gauge symmetry for each generation of quarks. After switching to the mass eigenstate basis, the coupling of the quarks to $Z^{\prime}$ can be written as $g^{\prime} Q_{i j} \bar{u}_{R}^{i} \gamma^{\mu} u_{R}^{j} Z_{\mu}^{\prime}$, where $Q_{i j}=Q_{k}^{\prime} V_{i k}^{R \dagger} V_{k j}^{R}$ and $V^{R, L}$ are the unitary matrices that diagonalise the quark mass matrix and $Q_{i}^{\prime}$ is the gauge charge of the quark of the $i^{\text {th }}$ generation under the $\mathrm{U}(1)^{\prime}$. In our model, we assumed that $g_{i 3}=g^{\prime} Q_{i 3}$, where $i=1,2$, are the dominant couplings.

Since we do not want to charge the left-handed quarks under $\mathrm{U}(1)^{\prime}$, there are two ways to render the SM Yukawa couplings gauge invariant. One way is to charge the Higgs boson. Since every generation has different charge $Q_{i}^{\prime}$, we would need to introduce a different Higgs boson (with charge $-Q_{i}^{\prime}$ ) for every generation. This leads to theories with extra Higgs doublets, discussed in the past in the context of the forward-backward asymmetry [90]. The second way is to use the Froggatt-Nielsen (FN) mechanism, i.e. to interpret the Yukawa coupling as the expectation value of a dynamical scalar field $\phi$ that is charged under $\mathrm{U}(1)^{\prime}$. Either way, the construction of the flavour-changing $Z^{\prime}$ coupling requires extra scalars that are charged under $\mathrm{U}(1)^{\prime}$, either extra Higgs doublets or an extra FN type scalar. In our work we focused on model-independent aspects of the top flavourchanging DM model by assuming that these extra states are heavy enough so as not to play a role in LHC or DM detection experiments.

As for the second point, the model per se is anomalous. Charging only the right-handed quarks under the $\mathrm{U}(1)^{\prime}$ introduces gauge anomalies from triangle diagrams that involve the $Z^{\prime}$ and SM gauge bosons. Phenomenological and theoretical aspects of anomalous $\mathrm{U}(1)^{\prime}$ extensions of the SM have been extensively discussed, see [91-93] and references therein. In order to cancel the anomalies, new chiral fermions $\psi_{L, R}$ need to be added that are also charged under $\mathrm{U}(1)^{\prime}$ and the SM gauge groups. These chiral fermions get their mass by the spontaneous breaking of the $\mathrm{U}(1)^{\prime}$ gauge symmetry, via Yukawa interactions of type $y \varphi \bar{\psi}_{L} \psi_{R}$, so that $m_{\psi} \sim y v_{\varphi}$ while $m_{Z^{\prime}}=g^{\prime} v_{\varphi} / 2$. Therefore, for moderate $g_{i 3}$ and for typical $\mathrm{U}(1)^{\prime}$ charge assignments we expect that the mass of these fermions is not much heavier than $m_{Z^{\prime}}$, however leaving enough room to consider these extra states beyond the reach of LHC for large Yukawa couplings. Indeed, in our phenomenological analysis we 
take $g_{i 3}=0.6$ which suggests that the extra fermion masses can be easily larger than the $\mathrm{TeV}$ scale, which is e.g. beyond the current bound on heavy quarks of 950 (782) GeV from the ATLAS [94] (CMS [95]) experiments. Furthermore, in our analysis we assumed that the $Z / Z^{\prime}$ kinetic mixing is negligible. This term could be typically induced via radiative effects and its size depends on the specific UV completion of the model. We expect that with loop suppression and for $m_{Z^{\prime}} \gtrsim 400 \mathrm{GeV}$ there are no bounds from current direct search experiments [92], leaving a detailed investigation to future work.

Summarizing, in our work we have focused on model-independent aspects of the top flavour-changing DM model and neglected extra states related to possible UV completions by assuming that they are heavy enough to not affect the phenomenology significantly. It would be interesting to study the signatures of these states and obtain combined constraints by associating it with the analyses we performed.

\section{Acknowledgments}

The authors would like to thank fruitful discussions with P. Anastasopoulos, L. Calibbi, E. Dudas, F. Maltoni and B. Zaldivar. This work is supported in part by Vrije Universiteit Brussel through the Strategic Research Program 'High-Energy Physics', and in part by the Belgian Federal Science Policy Office through the Inter-university Attraction Pole P7/37. A.M. and P.T. are also supported in part by FWO-Vlaanderen through project G011410N. A.M. aknowledges the Pegasus FWO postdoctoral Fellowship. K.M. is supported by the "Theory-LHC-France Initiative" of CNRS (INP/IN2P3). S.M. is a Aspirant van het Fonds Wetenschappelijk Onderzoek - Vlaanderen.

Open Access. This article is distributed under the terms of the Creative Commons Attribution License (CC-BY 4.0), which permits any use, distribution and reproduction in any medium, provided the original author(s) and source are credited.

\section{References}

[1] D. Abercrombie et al., Dark Matter Benchmark Models for Early LHC Run-2 Searches: Report of the ATLAS/CMS Dark Matter Forum, arXiv:1507.00966 [INSPIRE].

[2] S. Jung, H. Murayama, A. Pierce and J.D. Wells, Top quark forward-backward asymmetry from new t-channel physics, Phys. Rev. D 81 (2010) 015004 [arXiv:0907.4112] [INSPIRE].

[3] J. Andrea, B. Fuks and F. Maltoni, Monotops at the LHC, Phys. Rev. D 84 (2011) 074025 [arXiv:1106.6199] [INSPIRE].

[4] I. Boucheneb, G. Cacciapaglia, A. Deandrea and B. Fuks, Revisiting monotop production at the LHC, JHEP 01 (2015) 017 [arXiv: 1407.7529] [INSPIRE].

[5] A. Kumar and S. Tulin, Top-flavored dark matter and the forward-backward asymmetry, Phys. Rev. D 87 (2013) 095006 [arXiv: 1303.0332] [INSPIRE].

[6] L. Lopez-Honorez and L. Merlo, Dark matter within the minimal flavour violation ansatz, Phys. Lett. B 722 (2013) 135 [arXiv:1303.1087] [InSPIRE]. 
[7] J. Kile and A. Soni, Flavored Dark Matter in Direct Detection Experiments and at LHC, Phys. Rev. D 84 (2011) 035016 [arXiv: 1104.5239] [InSPIRE].

[8] B. Batell, J. Pradler and M. Spannowsky, Dark Matter from Minimal Flavor Violation, JHEP 08 (2011) 038 [arXiv: 1105.1781] [INSPIRE].

[9] J.F. Kamenik and J. Zupan, Discovering Dark Matter Through Flavor Violation at the LHC, Phys. Rev. D 84 (2011) 111502 [arXiv:1107.0623] [INSPIRE].

[10] P. Agrawal, S. Blanchet, Z. Chacko and C. Kilic, Flavored Dark Matter and Its Implications for Direct Detection and Colliders, Phys. Rev. D 86 (2012) 055002 [arXiv:1109.3516] [INSPIRE].

[11] P. Agrawal, B. Batell, D. Hooper and T. Lin, Flavored Dark Matter and the Galactic Center Gamma-Ray Excess, Phys. Rev. D 90 (2014) 063512 [arXiv:1404.1373] [InSPIRE].

[12] P. Agrawal, M. Blanke and K. Gemmler, Flavored dark matter beyond Minimal Flavor Violation, JHEP 10 (2014) 72 [arXiv:1405.6709] [INSPIRE].

[13] L. Calibbi, A. Crivellin and B. Zaldívar, Flavor portal to dark matter, Phys. Rev. D 92 (2015) 016004 [arXiv: 1501.07268] [INSPIRE].

[14] F. Bishara, A. Greljo, J.F. Kamenik, E. Stamou and J. Zupan, Dark Matter and Gauged Flavor Symmetries, JHEP 12 (2015) 130 [arXiv:1505.03862] [INSPIRE].

[15] K. Cheung, K. Mawatari, E. Senaha, P.-Y. Tseng and T.-C. Yuan, The Top Window for dark matter, JHEP 10 (2010) 081 [arXiv: 1009.0618] [INSPIRE].

[16] U. Haisch, F. Kahlhoefer and J. Unwin, The impact of heavy-quark loops on LHC dark matter searches, JHEP 07 (2013) 125 [arXiv: 1208.4605] [INSPIRE].

[17] T. Lin, E.W. Kolb and L.-T. Wang, Probing dark matter couplings to top and bottom quarks at the LHC, Phys. Rev. D 88 (2013) 063510 [arXiv:1303.6638] [InSPIRE].

[18] ATLAS collaboration, Search for dark matter in events with heavy quarks and missing transverse momentum in pp collisions with the ATLAS detector, Eur. Phys. J. C 75 (2015) 92 [arXiv: 1410.4031] [INSPIRE].

[19] CMS collaboration, Search for the production of dark matter in association with top-quark pairs in the single-lepton final state in proton-proton collisions at $\sqrt{s}=8 \mathrm{TeV}$, JHEP 06 (2015) 121 [arXiv: 1504.03198] [INSPIRE].

[20] M.R. Buckley, D. Feld and D. Goncalves, Scalar Simplified Models for Dark Matter, Phys. Rev. D 91 (2015) 015017 [arXiv: 1410.6497] [INSPIRE].

[21] U. Haisch and E. Re, Simplified dark matter top-quark interactions at the LHC, JHEP 06 (2015) 078 [arXiv: 1503.00691] [InSPIRE].

[22] O. Mattelaer and E. Vryonidou, Dark matter production through loop-induced processes at the LHC: the s-channel mediator case, Eur. Phys. J. C 75 (2015) 436 [arXiv:1508.00564] [INSPIRE].

[23] M. Backović, M. Krämer, F. Maltoni, A. Martini, K. Mawatari and M. Pellen, Higher-order QCD predictions for dark matter production at the LHC in simplified models with s-channel mediators, Eur. Phys. J. C 75 (2015) 482 [arXiv: 1508.05327] [InSPIRE].

[24] B. Fuks, P. Richardson and A. Wilcock, Studying the sensitivity of monotop probes to compressed supersymmetric scenarios at the LHC, Eur. Phys. J. C 75 (2015) 308 [arXiv: 1408.3634] [INSPIRE]. 
[25] ATLAS collaboration, Search for invisible particles produced in association with single-top-quarks in proton-proton collisions at $\sqrt{s}=8 \mathrm{TeV}$ with the ATLAS detector, Eur. Phys. J. C 75 (2015) 79 [arXiv:1410.5404] [InSPIRE].

[26] CMS collaboration, Search for Monotop Signatures in Proton-Proton Collisions at $\sqrt{s}=8$ TeV, Phys. Rev. Lett. 114 (2015) 101801 [arXiv:1410.1149] [INSPIRE].

[27] J. Wang, C.S. Li, D.Y. Shao and H. Zhang, Search for the signal of monotop production at the early LHC, Phys. Rev. D 86 (2012) 034008 [arXiv:1109.5963] [INSPIRE].

[28] A. Kumar, J.N. Ng, A. Spray and P.T. Winslow, Tracking down the top quark forward-backward asymmetry with monotops, Phys. Rev. D 88 (2013) 075012 [arXiv: 1308.3712] [INSPIRE].

[29] E. Alvarez, E.C. Leskow, J. Drobnak and J.F. Kamenik, Leptonic Monotops at LHC, Phys. Rev. D 89 (2014) 014016 [arXiv: 1310.7600] [INSPIRE].

[30] J.-L. Agram, J. Andrea, M. Buttignol, E. Conte and B. Fuks, Monotop phenomenology at the Large Hadron Collider, Phys. Rev. D 89 (2014) 014028 [arXiv:1311.6478] [InSPIRE].

[31] J.N. Ng and A. de la Puente, Probing Radiative Neutrino Mass Generation through Monotop Production, Phys. Rev. D 90 (2014) 095018 [arXiv:1404.1415] [INSPIRE].

[32] R. Allahverdi, M. Dalchenko, B. Dutta, Y. Gao and T. Kamon, Distinguishing Standard Model Extensions using Monotop Chirality at the LHC, arXiv:1507.02271 [INSPIRE].

[33] A. Rajaraman, J. Smolinsky and P. Tanedo, On-Shell Mediators and Top-Charm Dark Matter Models for the Fermi-LAT Galactic Center Excess, arXiv:1503.05919 [INSPIRE].

[34] D. Hooper and L. Goodenough, Dark Matter Annihilation in The Galactic Center As Seen by the Fermi Gamma Ray Space Telescope, Phys. Lett. B 697 (2011) 412 [arXiv:1010.2752] [INSPIRE].

[35] S. Murgia, Observation of the high energy gamma-ray emission towards the galactic center, Fifth FERMI Symposium, Nagoya Japan, 20-24 October 2014.

[36] Fermi-LAT collaboration, T.A. Porter and S. Murgia, Observations of High-Energy Gamma-Ray Emission Toward the Galactic Centre with the Fermi Large Area Telescope, arXiv: 1507.04688 [INSPIRE].

[37] R. Bartels, S. Krishnamurthy and C. Weniger, Strong support for the millisecond pulsar origin of the Galactic center GeV excess, Phys. Rev. Lett. 116 (2016) 051102 [arXiv: 1506.05104] [INSPIRE].

[38] S.K. Lee, M. Lisanti, B.R. Safdi, T.R. Slatyer and W. Xue, Evidence for Unresolved Gamma-Ray Point Sources in the Inner Galaxy, Phys. Rev. Lett. 116 (2016) 051103 [arXiv: 1506.05124] [INSPIRE].

[39] D. Gaggero, M. Taoso, A. Urbano, M. Valli and P. Ullio, Towards a realistic astrophysical interpretation of the gamma-ray Galactic center excess, JCAP 12 (2015) 056 [arXiv: 1507.06129] [INSPIRE].

[40] K.N. Abazajian and R.E. Keeley, A Bright Gamma-ray Galactic Center Excess and Dark Dwarfs: Strong Tension for Dark Matter Annihilation Despite Milky Way Halo Profile and Diffuse Emission Uncertainties, arXiv:1510.06424 [INSPIRE].

[41] L.-B. Jia, Search for pseudoscalar-mediated WIMPs in $t \rightarrow c$ transitions with missing energy, Phys. Rev. D 92 (2015) 074006 [arXiv: 1506. 05293] [INSPIRE]. 
[42] Q.-H. Cao, D. McKeen, J.L. Rosner, G. Shaughnessy and C.E.M. Wagner, Forward-Backward Asymmetry of Top Quark Pair Production, Phys. Rev. D 81 (2010) 114004 [arXiv: 1003.3461] [INSPIRE].

[43] M. Ciuchini, E. Franco, D. Guadagnoli, V. Lubicz, M. Pierini, V. Porretti et al., D- $\bar{D}$ mixing and new physics: General considerations and constraints on the MSSM, Phys. Lett. $\mathbf{B} 655$ (2007) 162 [hep-ph/0703204] [INSPIRE].

[44] D0 collaboration, V.M. Abazov et al., An Improved determination of the width of the top quark, Phys. Rev. D 85 (2012) 091104 [arXiv:1201.4156] [INSPIRE].

[45] CDF collaboration, T.A. Aaltonen et al., Direct Measurement of the Total Decay Width of the Top Quark, Phys. Rev. Lett. 111 (2013) 202001 [arXiv:1308.4050] [INSPIRE].

[46] CMS collaboration, Search for Flavor-Changing Neutral Currents in Top-Quark Decays $t \rightarrow Z q$ in pp Collisions at $\sqrt{s}=8$ TeV, Phys. Rev. Lett. $112(2014) 171802$ [arXiv: 1312.4194] [INSPIRE].

[47] J. Alwall et al., Comparative study of various algorithms for the merging of parton showers and matrix elements in hadronic collisions, Eur. Phys. J. C 53 (2008) 473 [arXiv:0706.2569] [INSPIRE].

[48] CDF collaboration, T. Aaltonen et al., Evidence for a Mass Dependent Forward-Backward Asymmetry in Top Quark Pair Production, Phys. Rev. D 83 (2011) 112003 [arXiv: 1101.0034] [INSPIRE].

[49] D0 collaboration, V.M. Abazov et al., Forward-backward asymmetry in top quark-antiquark production, Phys. Rev. D 84 (2011) 112005 [arXiv: 1107.4995] [INSPIRE].

[50] J. Cao, L. Wang, L. Wu and J.M. Yang, Top quark forward-backward asymmetry, FCNC decays and like-sign pair production as a joint probe of new physics, Phys. Rev. D 84 (2011) 074001 [arXiv: 1101.4456] [INSPIRE].

[51] E.L. Berger, Q.-H. Cao, C.-R. Chen, C.S. Li and H. Zhang, Top Quark Forward-Backward Asymmetry and Same-Sign Top Quark Pairs, Phys. Rev. Lett. 106 (2011) 201801 [arXiv:1101.5625] [INSPIRE].

[52] CMS collaboration, Search for Same-Sign Top-Quark Pair Production at $\sqrt{s}=7$ TeV and Limits on Flavour Changing Neutral Currents in the Top Sector, JHEP 08 (2011) 005 [arXiv:1106.2142] [INSPIRE].

[53] ATLAS collaboration, Search for same-sign top-quark production and fourth-generation down-type quarks in pp collisions at $\sqrt{s}=7$ TeV with the ATLAS detector, JHEP 04 (2012) 069 [arXiv: 1202.5520] [INSPIRE].

[54] S. Jung, A. Pierce and J.D. Wells, Top quark asymmetry from a non-Abelian horizontal symmetry, Phys. Rev. D 83 (2011) 114039 [arXiv:1103.4835] [INSPIRE].

[55] J. Goodman, M. Ibe, A. Rajaraman, W. Shepherd, T.M.P. Tait and H.-B. Yu, Constraints on Light Majorana dark Matter from Colliders, Phys. Lett. B 695 (2011) 185 [arXiv: 1005.1286] [INSPIRE].

[56] Y. Bai, P.J. Fox and R. Harnik, The Tevatron at the Frontier of Dark Matter Direct Detection, JHEP 12 (2010) 048 [arXiv: 1005.3797] [INSPIRE].

[57] P.J. Fox, R. Harnik, J. Kopp and Y. Tsai, Missing Energy Signatures of Dark Matter at the LHC, Phys. Rev. D 85 (2012) 056011 [arXiv:1109.4398] [InSPIRE]. 
[58] I.M. Shoemaker and L. Vecchi, Unitarity and Monojet Bounds on Models for DAMA, CoGeNT and CRESST-II, Phys. Rev. D 86 (2012) 015023 [arXiv:1112.5457] [INSPIRE].

[59] G. Busoni, A. De Simone, E. Morgante and A. Riotto, On the Validity of the Effective Field Theory for Dark Matter Searches at the LHC, Phys. Lett. B 728 (2014) 412 [arXiv: 1307.2253] [INSPIRE].

[60] O. Buchmueller, M.J. Dolan and C. McCabe, Beyond Effective Field Theory for Dark Matter Searches at the LHC, JHEP 01 (2014) 025 [arXiv: 1308.6799] [INSPIRE].

[61] N.D. Christensen et al., A Comprehensive approach to new physics simulations, Eur. Phys. J. C 71 (2011) 1541 [arXiv:0906.2474] [INSPIRE].

[62] A. Alloul, N.D. Christensen, C. Degrande, C. Duhr and B. Fuks, FeynRules 2.0 - A complete toolbox for tree-level phenomenology, Comput. Phys. Commun. 185 (2014) 2250 [arXiv: 1310.1921] [INSPIRE].

[63] C. Degrande, C. Duhr, B. Fuks, D. Grellscheid, O. Mattelaer and T. Reiter, UFO - The Universal FeynRules Output, Comput. Phys. Commun. 183 (2012) 1201 [arXiv:1108.2040] [INSPIRE].

[64] P. de Aquino, W. Link, F. Maltoni, O. Mattelaer and T. Stelzer, ALOHA: Automatic Libraries Of Helicity Amplitudes for Feynman Diagram Computations, Comput. Phys. Commun. 183 (2012) 2254 [arXiv:1108.2041] [INSPIRE].

[65] J. Alwall et al., The automated computation of tree-level and next-to-leading order differential cross sections and their matching to parton shower simulations, JHEP 07 (2014) 079 [arXiv: 1405.0301] [INSPIRE].

[66] G. Bélanger, F. Boudjema, A. Pukhov and A. Semenov, MicrOMEGAs 2.0: A Program to calculate the relic density of dark matter in a generic model, Comput. Phys. Commun. 176 (2007) 367 [hep-ph/0607059] [INSPIRE].

[67] G. Bélanger, F. Boudjema, A. Pukhov and A. Semenov, Dark matter direct detection rate in a generic model with MicrOMEGAs 2.2, Comput. Phys. Commun. 180 (2009) 747 [arXiv:0803.2360] [INSPIRE].

[68] M. Backovic, K. Kong and M. McCaskey, MadDM v.1.0: Computation of Dark Matter Relic Abundance Using MadGraph5, Physics of the Dark Universe 5-6 (2014) 18 [arXiv: 1308.4955] [INSPIRE].

[69] M. Backović, A. Martini, O. Mattelaer, K. Kong and G. Mohlabeng, Direct Detection of Dark Matter with MadDM v.2.0, Phys. Dark Univ. 9-10 (2015) 37 [arXiv: 1505. 04190] [INSPIRE].

[70] T. Sjöstrand, S. Mrenna and P.Z. Skands, PYTHIA 6.4 Physics and Manual, JHEP 05 (2006) 026 [hep-ph/0603175] [INSPIRE].

[71] J. Alwall, S. de Visscher and F. Maltoni, QCD radiation in the production of heavy colored particles at the LHC, JHEP 02 (2009) 017 [arXiv: 0810.5350] [INSPIRE].

[72] DELPHES 3 collaboration, J. de Favereau et al., DELPHES 3, A modular framework for fast simulation of a generic collider experiment, JHEP 02 (2014) 057 [arXiv:1307.6346] [INSPIRE].

[73] E. Conte, B. Fuks and G. Serret, MadAnalysis 5, A User-Friendly Framework for Collider Phenomenology, Comput. Phys. Commun. 184 (2013) 222 [arXiv:1206.1599] [INSPIRE]. 
[74] E. Conte, B. Dumont, B. Fuks and C. Wymant, Designing and recasting LHC analyses with MadAnalysis 5, Eur. Phys. J. C 74 (2014) 3103 [arXiv:1405.3982] [INSPIRE].

[75] https://twiki.cern.ch/twiki/bin/view/LHCPhysics/LHCTopWG.

[76] M. Cacciari, G.P. Salam and G. Soyez, The Anti-k(t) jet clustering algorithm, JHEP 04 (2008) 063 [arXiv: 0802.1189] [INSPIRE].

[77] CMS collaboration, Identification of b-quark jets with the CMS experiment, 2013 JINST 8 P04013 [arXiv: 1211. 4462] [INSPIRE].

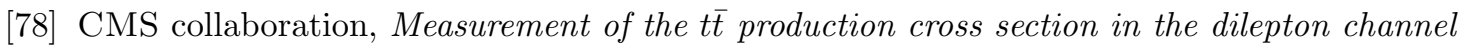
in pp collisions at $\sqrt{s}=8 \mathrm{TeV}$, JHEP 02 (2014) 024 [Erratum ibid. 1402 (2014) 102] [arXiv: 1312.7582] [INSPIRE].

[79] ATLAS collaboration, Performance and Calibration of the JetFitterCharm Algorithm for c-Jet Identification, ATL-PHYS-PUB-2015-001 (2015).

[80] Planck collaboration, P.A.R. Ade et al., Planck 2013 results. XVI. Cosmological parameters, Astron. Astrophys. 571 (2014) A16 [arXiv:1303.5076] [INSPIRE].

[81] Fermi-LAT collaboration, M. Ackermann et al., Searching for Dark Matter Annihilation from Milky Way Dwarf Spheroidal Galaxies with Six Years of Fermi Large Area Telescope Data, Phys. Rev. Lett. 115 (2015) 231301 [arXiv:1503.02641] [INSPIRE].

[82] M. Cirelli et al., PPPC 4 DM ID: A Poor Particle Physicist Cookbook for Dark Matter Indirect Detection, JCAP 03 (2011) 051 [Erratum ibid. 1210 (2012) E01] [arXiv: 1012.4515] [INSPIRE].

[83] AMS-02 collaboration, AMS Days at CERN, Geneva Switzerland, 15-17 April 2015.

[84] G. Giesen et al., AMS-02 antiprotons, at last! Secondary astrophysical component and immediate implications for Dark Matter, JCAP 09 (2015) 023 [arXiv:1504.04276] [INSPIRE].

[85] H.-B. Jin, Y.-L. Wu and Y.-F. Zhou, Upper limits on dark matter annihilation cross sections from the first AMS-02 antiproton data, Phys. Rev. D 92 (2015) 055027 [arXiv:1504.04604] [INSPIRE].

[86] R. Kappl, A. Reinert and M.W. Winkler, AMS-02 Antiprotons Reloaded, JCAP 10 (2015) 034 [arXiv: 1506.04145] [INSPIRE].

[87] A. Berlin, D. Hooper and S.D. McDermott, Simplified Dark Matter Models for the Galactic Center Gamma-Ray Excess, Phys. Rev. D 89 (2014) 115022 [arXiv:1404.0022] [InSPIRE].

[88] A. Alves, S. Profumo, F.S. Queiroz and W. Shepherd, Effective field theory approach to the Galactic Center gamma-ray excess, Phys. Rev. D 90 (2014) 115003 [arXiv:1403.5027] [INSPIRE].

[89] F. Kahlhoefer, K. Schmidt-Hoberg, T. Schwetz and S. Vogl, Implications of unitarity and gauge invariance for simplified dark matter models, JHEP 02 (2016) 016 [arXiv: 1510.02110] [INSPIRE].

[90] P. Ko, Y. Omura and C. Yu, Top Forward-Backward Asymmetry and the CDF Wjj Excess in Leptophobic U(1)' Flavor Models, Phys. Rev. D 85 (2012) 115010 [arXiv:1108.0350] [INSPIRE].

[91] P. Anastasopoulos, M. Bianchi, E. Dudas and E. Kiritsis, Anomalies, anomalous U(1)'s and generalized Chern-Simons terms, JHEP 11 (2006) 057 [hep-th/0605225] [INSPIRE]. 
[92] Y. Mambrini, The ZZ' kinetic mixing in the light of the recent direct and indirect dark matter searches, JCAP 07 (2011) 009 [arXiv: 1104.4799] [INSPIRE].

[93] E. Dudas, L. Heurtier, Y. Mambrini and B. Zaldivar, Extra U(1), effective operators, anomalies and dark matter, JHEP 11 (2013) 083 [arXiv:1307.0005] [INSPIRE].

[94] ATLAS collaboration, Search for production of vector-like quark pairs and of four top quarks in the lepton-plus-jets final state in pp collisions at $\sqrt{s}=8 \mathrm{TeV}$ with the ATLAS detector, JHEP 08 (2015) 105 [arXiv: 1505.04306] [INSPIRE].

[95] CMS collaboration, Inclusive search for a vector-like $T$ quark with charge $\frac{2}{3}$ in pp collisions at $\sqrt{s}=8 \mathrm{TeV}$, Phys. Lett. B 729 (2014) 149 [arXiv:1311.7667] [InSPIRE]. 